\title{
Scattering-based focusing for imaging in highly complex media from band-limited, multi-component data
}

\author{
David Vargas ${ }^{1}$, Ivan Vasconcelos ${ }^{1}$, Yanadet Sripanich ${ }^{2}$, Matteo Ravasi $^{3}$, \\ 1 Department of Earth Sciences, Utrecht University, 3584 CB, The \\ Netherlands, \\ 2 PTT Exploration and Production Public Company Limited, Chatuchak, \\ Bangkok 10900 Thailand, \\ 3 Earth Science and Engineering, King Abdullah University of Science and \\ Technology, Thuwal 23955, Kingdom of Saudi Arabia.
}

(May 20, 2021)

GEO-2020-0939

Running head: Scattering-based Marchenko

\begin{abstract}
Reconstructing the details of subsurface structures deep beneath complex overburden structures, such as sub-salt, remains a challenge for seismic imaging. Over the past years, the Marchenko redatuming approach has proven to reliably retrieve full-wavefield information in the presence of complex overburden effects. When used for redatuming, current practical Marchenko schemes cannot make use of a priori subsurface models with sharp contrasts because of their requirements regarding initial focusing functions, which for sufficiently complex media can result in redatumed fields with significant waveform inaccuracies. Using a scattering framework, we present an alternative form of the Marchenko representation
\end{abstract}


that aims at retrieving only the unknown perturbations to both focusing functions and redatumed fields. From this framework, we propose a two-step practical focusing-based redatuming scheme that first solves an inverse problem for the background focusing functions, which are then used to estimate the perturbations to focusing functions and redatumed fields. In our scheme, initial focusing functions are significantly different from previous approaches since they contain complex waveforms encoding the full transmission response of the a priori model. Our goal is the handling of not only highly complex media but also realistic data - band-limited, unevenly sampled, free-surface-multiple contaminated data. To that end, we combine the versatility of Rayleigh-Marchenko redatuming with the proposed scattering-based scheme allowing an extended version of the method able to handle single-sided band-limited multicomponent data. This Scattering-Rayleigh-Marchenko strategy accurately retrieves wavefields while requiring minimum pre-processing of the data. In support of the new methods, we present a comprehensive set of numerical tests using a complex 2D subsalt model. Our numerical results show that the scattering approaches retrieve accurate redatumed fields that appropriately account for the complexity of the a priori model. We show that the improvements in wavefield retrieval translate into measurable improvements in our subsalt images. 


\section{INTRODUCTION}

Accurate estimation of the stratigraphy and properties of complex subsurface geology has long represented a challenge for seismic imaging, especially in the presence of salt (or basalt) formations in the overburden (Leveille et al., 2011). This is largely due to uneven illumination of the target area arising from the complex propagation in the overburden as well as the presence of strong reverberations in the recorded surface data (Jones and Davison, 2014). On one hand, advances in seismic acquisition techniques, such as the adoption wide-azimuth surveys, have played a major role in providing more even subsurface illumination, which in turn can help reduce the ill-posed nature of the associated imaging problem. On the other hand, it is only by incorporating better physics in the imaging process that the full potential of the recorded seismic data can be exploited. This is for example justified by the uplift in the quality and focusing of reflectors provided by high-end, wave equation-based migration methods such as reverse-time migration (McMechan, 1983; Baysal et al., 1983; Farmer et al., 2009), data or image domain least-squares migration (Nemeth et al., 1999; Fletcher et al., 2016; Arasanipalai et al., 2019) as well as Full Waveform Inversion (Tarantola, 1984; Virieux and Operto, 2009; Esser et al., 2016; Wang et al., 2019). Moreover, a variety of approaches, emerged under the pursuit of imaging with multiples, have also shown their effectiveness in such settings; these methods turn multiple reverberations originating from high impedance contrasts around salt and basalt structures into useful signal that can complement the illumination of primaries (Malcolm et al., 2008; Liu et al., 2015).

Alongside model-driven approaches that aim at iteratively reconstructing the velocity (and/or reflectivity) model of the subsurface, from which a more accurate propagation of the underlying wavefield can be numerically modeled, a great deal of research has been 
recently devoted to target-oriented methods. By targeting a specific area of the subsurface, these methods promise to provide reservoir-characterisation-ready models of the subsurface (da Costa et al., 2019; Cui et al., 2020; Guo and Alkhalifah, 2020). The reduced computational domain and size of the model to invert for, can allow for the inclusions of higher frequencies and even more complex physics (e.g., elastic, attenuation) in the modeling operator used to describe the data. Nevertheless, the quality of the final image and/or inverted properties is strongly dependent on the ability to accurately redatum surface data at the target level of interest. To this regard, novel wavefield extrapolation techniques that go beyond the single-scattering (i.e., Born) approximation open up new ways to create such target data.

Marchenko redatuming (Wapenaar et al., 2014b; van der Neut et al., 2015b) has recently been demonstrated to be a reliable method for retrieving full-wavefield subsurface responses in a variety of geological settings which can accommodate moderately complex overburden geology. The Marchenko method makes use of the recorded reflection response and an estimate of the direct transmission response in the medium to estimate so-called focusing functions, that serve as operators to focus energy at specific points in the subsurface. Once the redatuming step has been performed, the resulting subsurface wavefields can be used to to create artefact-free images of the subsurface (Wapenaar et al., 2014b) or estimate local properties (Cui et al., 2020) by naturally including all order of multiples present in seismic reflection data. From this perspective, Marchenko redatuming represents an ideal platform for creating subsurface wavefield suitable for target-oriented imaging and inversion beneath complex structures such as salt, but also other environments including sub-basalt or other highly complex tectonic settings.

To that end, however, the original Marchenko scheme has strict requirements with regards to the reflection response. Namely, it requires large aperture acquisition geometries, it should 
represent data from a surface-multiple-free survey, and have flat, very broadband frequency content. When it comes to practical implementation of the method, the requirement for an accurate deconvolution of the source wavelet, removal of surface-related multiples, and source/receiver co-location hinders its application to real data sets. Moreover, treating surface-related multiples as noise that must be removed from the data is not only technically challenging and time consuming, but it may also not be beneficial in complex media. Surface multiples can carry complementary information compared to primaries since, when compared to surface-multiple-free data, they are exposed to longer propagation times and possibly different propagation paths in the subsurface as a consequence of the natural interactions of finite-aperture data with a free surface in a highly complex medium. Furthermore, on the subject of data pre-processing for Marchenko redatuming, source deconvolution with an erroneously estimated source wavelet leads to strong coherent artefacts in the local images (Mildner et al., 2019b). In many ways, the success of single-sided Green's functions retrieval depends upon the availability of accurate focusing functions. Dukalski et al. (2019) describe how to correct for the effect of short-period multiples in the Marchenko framework, by adding energy conservation constraints to the focusing problem. To relax on the originally strict acquisition requirements, Rayleigh-Marchenko redatuming (R-Marchenko) was introduced by Ravasi (2017) and Slob and Wapenaar (2017). The main advantage of R-Marchenko is the use of a band-limited operator defined in terms of vertical particle velocity rather than a broadband reflection response, as is the case in standard Marchenko implementations. Such consideration significantly reduces the need for most preprocessing steps.

More importantly in the context of this work, previous versions of the Marchenko scheme could not accommodate highly complex media such as subsalt - particularly in the presence of an a priori model containing sharp contrasts. This is because currently available Marchenko 
schemes explicitly require an estimate of a single direct arrival from a chosen focal point to the survey surface. In most approaches so far, this estimation is either based on initial smooth velocity model (no sharp contrasts), or by selecting/windowing first arrivals obtained by modeling, thus not being able to fully utilize complete waveform information contained in the modeling through complex velocity models with high (or low) acoustic impedance inclusions, such as in the case of salt bodies. Despite initial reports of successful attempts imaging complex subsalt structures (Staring et al., 2018; Jia et al., 2018; Staring and Wapenaar, 2020), Vasconcelos et al. (2014) show that the accuracy of conventional Marchenko redatuming can be considerably lower when dealing with highly complex media. To better reproduce seismic subsurface responses in such conditions, Vasconcelos and Sripanich (2019) propose a modified Marchenko scheme that can incorporate available information from previously estimated, complex subsurface models by introducing an auxiliary Marchenko system of equations for a reference model as a constraint to the solution in the true medium.

In this paper, we first build on the work of Vasconcelos and Sripanich (2019) and present a scattering-based Marchenko framework designed to handle highly-complex media, while making full use of state-of-the-art velocity models with sharp contrasts. To account for band-limited data in the presence of free-surface multiples and with realistic acquisition geometries, we combine the versatility of R-Marchenko with the scattering-based Marchenko's ability to handle redatuming in complex media. This enables us to derive a new scatteringbased Rayleigh-Marchenko method - SR-Marchenko - that shows a data-robust, superior performance to previously developed approaches in highly complex media. The new strategy naturally accounts for the compatibility between real and modelled data in the perturbation operator, which in turn allows for rather direct application on field multicomponent data sets. To evaluate the effectiveness of the new method, we present a comprehensive set of 
numerical tests using a complex 2D susbalt model. The focusing and Green's functions produced by the SR-Marchenko, R-Marchenko and the original Marchenko schemes are compared against each other as well as against a numerically modeled pressure field. Such wavefields are further used as input for a step of source redatuming via multi-dimensional deconvolution followed by localized imaging by means of reverse-time migration. At all stages of redatuming, we observe that the wavefields retrieved from our new approach are superior in terms of both events continuity and amplitude fidelity, suggesting that wavefield redatuming and imaging in complex media by means of Marchenko equations can greatly benefit from further information on the underlying subsurface media, i.e, migration velocity models.

\section{BROADBAND WAVEFIELD EXTRAPOLATION}

In this section, we review the main aspects involved in the traditional implementations of single-sided wavefield focusing and extrapolation, followed by an extension of Marchenko redatuming accounting for scattering effects in connection with a priori reference model. Contrary to the current practice of approximating the initial focusing function by a timereversed direct arrival, this strategy offers the possibility of estimating a reference-medium inverse transmission response, accounting for the effects of geometrical spreading and the kinematics of primaries as well as internal multiples in the given model. This more accurate focusing approach positively impacts the subsequent redatuming step, which in turn benefits the resulting imaging process. Estimating the full transmission response is, however, sensitive to potential inaccuracies in the reference model. Such errors may lead to a time shift in the retrieved focusing functions as well as the arising of artefacts, which ultimately influence the quality of the retrieved Green's functions (Broggini et al., 2014; Thorbecke et al., 2013). 
Further analysis of this subject and its implications for redatuming are addressed in the discussion.

\section{Marchenko Focusing and Redatuming}

One-way reciprocity theorems (Wapenaar and Grimbergen, 1996) provide the basis to estimate Green's functions, $g\left(\mathbf{x}_{\mathbf{r}}, \mathbf{x}_{\mathbf{f}} ; t\right)$, from a virtual source, $\mathbf{x}_{\mathbf{f}}$, at a depth level $\partial \mathbb{D}_{i}$ to a set of receivers $\mathbf{x}_{\mathbf{r}}$ located along the surface $\partial \mathbb{D}_{0}$ using a single-sided focusing operator, conventionally called focusing function $f\left(\mathbf{x}_{\mathbf{r}}, \mathbf{x}_{\mathbf{f}} ; t\right)$ (Wapenaar et al., 2014a). Such a wavefield is defined in a reference configuration (i.e., a truncated medium) and collapses at zero time at a given depth level when injected from the surface. The original Marchenko framework is restricted to a lossless medium, in this case, supporting only acoustic waves, and does not consider evanescent wave modes at either the acquisition or focusing levels (Wapenaar et al., 2004). According to Wapenaar et al. (2014b), the Marchenko integral equations relate the focusing function in the truncated medium with the true medium's Green's function, using the measured reflection response as the kernel of a multidimensional convolution. In theory, the reflection response $R\left(\mathbf{x}_{\mathbf{r}}, \mathbf{x}_{\mathbf{r}}^{\prime} ; t\right)$ is a full-bandwidth field that does not include surface-related multiple effects and is, in general, assumed to be known. This procedure, in the frequency domain, is described by

$$
\begin{aligned}
g^{-}\left(\mathbf{x}_{\mathbf{f}}, \mathbf{x}_{\mathbf{r}}\right) & =\int_{\partial \mathbb{D}_{\mathbf{0}}} R\left(\mathbf{x}_{\mathbf{r}}, \mathbf{x}_{\mathbf{r}}^{\prime}\right) f^{+}\left(\mathbf{x}_{\mathbf{r}}^{\prime}, \mathbf{x}_{\mathbf{f}}\right) d \mathbf{x}_{\mathbf{r}}^{\prime}-f^{-}\left(\mathbf{x}_{\mathbf{r}}, \mathbf{x}_{\mathbf{f}}\right) \\
-g^{+*}\left(\mathbf{x}_{\mathbf{f}}, \mathbf{x}_{\mathbf{r}}\right) & =\int_{\partial \mathbb{D}_{\mathbf{0}}} R^{*}\left(\mathbf{x}_{\mathbf{r}}, \mathbf{x}_{\mathbf{r}}^{\prime}\right) f^{-}\left(\mathbf{x}_{\mathbf{r}}^{\prime}, \mathbf{x}_{\mathbf{f}}\right) d \mathbf{x}_{\mathbf{r}}^{\prime}-f^{+}\left(\mathbf{x}_{\mathbf{r}}, \mathbf{x}_{\mathbf{f}}\right),
\end{aligned}
$$

where the frequency dependence has been omitted for notational brevity. Here, * represents complex conjugation, i.e., time reversal in the time domain. A typical Marchenko setup consists of a set of sources, $\mathbf{x}_{\mathbf{r}}^{\prime}$, and receivers, $\mathbf{x}_{\mathbf{r}}$, collocated on a transparent surface 
$\partial \mathbb{D}_{0}$. Similarly, the virtual source location, $\mathbf{x}_{\mathbf{f}}$, lays on an arbitrary focusing depth level $\partial \mathbb{D}_{i}$. The required two-way response from $\mathbf{x}_{\mathbf{f}}$ to $\mathbf{x}_{\mathbf{r}}$ is given in terms of its up- and down-going components, $g^{-}$and $g^{+}$, according to $g=g^{-}+g^{+}$. On the other hand, the up- and downgoing focusing functions, $f^{ \pm}$, are defined in a truncated version of the medium designed to be reflection free outside the region between levels $\partial \mathbb{D}_{0}$ and $\partial \mathbb{D}_{i}$.

From a practical perspective, sources and receivers are positioned at discrete locations. Therefore, it is useful to discretize the Marchenko system of equations 1 and 2, and recast them in a compact matrix-vector notation. In the discrete Marchenko framework (van der Neut et al., 2015b), the reflection response acts as a multidimensional filter on the focusing operators returning the desired Green's functions, such that

$$
\left[\begin{array}{c}
-\mathbf{g}^{-} \\
\mathbf{g}^{+*}
\end{array}\right]=\left[\begin{array}{cc}
\mathbf{I} & -\mathbf{R} \\
-\mathbf{R}^{*} & \mathbf{I}
\end{array}\right]\left[\begin{array}{l}
\mathbf{f}^{-} \\
\mathbf{f}^{+}
\end{array}\right]
$$

denotes the discrete version of the Marchenko system. The Marchenko-operator matrix is composed by an identity matrix, $\mathbf{I}$, together with matrix operators $\mathbf{R}$ and $\mathbf{R}^{*}$ which apply multidimensional space-time convolution and correlation - note that this operator form applies to both time- and frequency-domain versions of the system, though of course the numerical structure of the operators themselves differ. As per focusing $\mathbf{f}^{ \pm}$and Green's $\mathbf{g}^{ \pm}$ functions, they are concatenated in block-vector form (i.e., array stacking) in time-space to form the vectors of the system. Given that equations 1 and 2 represent an underdetermined system of two equations with four unknown functions $\left(\mathbf{g}^{-}, \mathbf{g}^{+}, \mathbf{f}^{-}\right.$and $\left.\mathbf{f}^{+}\right)$, additional constraints need to be introduced in order to solve such a system. In the particular case of relatively smooth media with moderately curved interbeds and limited acquisition aperture, it is plausible to assume a causality argument stating that focusing and Green's functions lay on different space-time regions. Provided that this condition holds, $\mathbf{g}$ and $\mathbf{f}$ do not 
mutually interfere with one another (Wapenaar et al., 2014b). To numerically enforce causality, we implement a window matrix $\boldsymbol{\Psi}$ designed to be symmetric in time such that it removes all causal and acausal events within the time interval $-t_{d}\left(\mathbf{x}_{\mathbf{r}}, \mathbf{x}_{\mathbf{f}}\right) \leq t \leq t_{d}\left(\mathbf{x}_{\mathbf{r}}, \mathbf{x}_{\mathbf{f}}\right)$, i.e $\mathbf{\Psi} \mathbf{g}^{-}=\mathbf{0}$ and $\mathbf{\Psi} \mathbf{g}^{+}=\mathbf{0}$. On the other hand, the downgoing focusing function can be seen as the composition of a direct wave followed by a coda containing all different orders of scattering that arrive at later times, $\mathbf{f}^{+}=\mathbf{f}_{d}^{+}+\mathbf{f}_{m}^{+}$. The action of the window matrix on $\mathbf{f}^{+}$only removes its direct wave, i.e $\mathbf{\Psi} \mathbf{f}^{+}=\mathbf{f}_{m}^{+}$, and does not have any effect on its upgoing counterpart $\boldsymbol{\Psi} \mathbf{f}^{-}=\mathbf{f}^{-}$. In light of these arguments, the number of unknowns in equations 1 and 2 is reduced once $\boldsymbol{\Psi}$ is applied to both sides of the equations (van der Neut et al., 2015b). Finally, a simplified matrix-vector representation for the discrete case follows after rearranging the remaining terms,

$$
\left[\begin{array}{cc}
\mathbf{0} & \mathbf{\Psi R} \\
\mathbf{\Psi} \mathbf{R}^{*} & \mathbf{0}
\end{array}\right]\left[\begin{array}{c}
\mathbf{0} \\
\mathbf{f}_{d}^{+}
\end{array}\right]=\left[\begin{array}{cc}
\mathbf{I} & -\mathbf{\Psi R} \\
-\mathbf{\Psi} \mathbf{R}^{*} & \mathbf{I}
\end{array}\right]\left[\begin{array}{c}
\mathbf{f}^{-} \\
\mathbf{f}_{m}^{+}
\end{array}\right] .
$$

Evaluation of equation 4 requires the direct part of the downgoing focusing function $\left(\mathbf{f}_{d}^{+}\right)$ to be known. In principle, if the direct part of the transmission response in the truncated reference medium $\mathbf{T}_{d}$ is available, $\mathbf{f}_{d}^{+}$can be obtained from the focusing condition, $\mathbf{i}=\mathbf{T}_{d} \mathbf{f}_{d}^{+}$, by direct inversion (van der Neut et al., 2015b). Here, vector i represents a band-limited delta function at the focal point $\mathbf{x}_{\mathbf{f}}$, at time zero. From a practical standpoint, instead of inverting for the initial focusing function, one common choice is to approximate it by taking the time-reversal of the modeled transmitted direct Green's function, i.e., $\mathbf{f}_{d}^{+} \approx \mathbf{g}_{d}^{*}$. This approximation demands some caution since it mostly accounts for kinematics and does not properly account for more subtle amplitude effects such as geometrical spreading, turning waves, or attenuation (Wapenaar et al., 2014a). 


\section{Redatuming of scattered fields}

Estimating focusing and Green's functions from single-sided Marchenko redatuming has proven to be challenging in highly complex media. Although the performance of Marchenko redatuming compared to conventional reverse-time extrapolation has been shown to be superior, several reconstructed seismic events are missing for subsurface characterized by sharp impedance contrasts, boulders, dipping layers, or strong diffractors (Vasconcelos et al., 2014). A major drawback of this approach is that some difficulties arise when trying to reproduce relative and absolute amplitudes, as well as retrieving all possible scattered arrivals. On the other hand, in such environments, greater emphasis is generally placed on building a seismic velocity model, as an accurate knowledge of the velocity field becomes even more important for successful migration results. For example, high-end migration models that can capture valuable information regarding the salt's sharp structure, provide additional constraints that may be used for purposes other than migration itself. Provided that such models are available, our goal is to take full advantage of them to retrieve full-wave scattered fields for redatuming and imaging purposes. To that end, here we extend the Marchenko framework to reconstruct perturbed focusing functions with respect to a background medium, and as a result, scattered Green's functions are also retrieved.

Our derivation starts by considering two independent Marchenko integral systems in the matrix-vector form (equation 3), the former describing the relationship between focusing and broadband seismic responses in the true medium, and the latter providing the connections between corresponding fields in a given reference (migration) model,

$$
\left[\begin{array}{c}
-\mathbf{g}^{-} \\
\mathbf{g}^{+*}
\end{array}\right]=\left[\begin{array}{cc}
\mathbf{I} & -\mathbf{R} \\
-\mathbf{R}^{*} & \mathbf{I}
\end{array}\right]\left[\begin{array}{l}
\mathbf{f}^{-} \\
\mathbf{f}^{+}
\end{array}\right] \quad, \quad\left[\begin{array}{c}
-\mathbf{g}_{0^{-}} \\
\mathbf{g}^{+*}
\end{array}\right]=\left[\begin{array}{cc}
\mathbf{I} & -\mathbf{R}_{\mathbf{0}} \\
-\mathbf{R}_{\mathbf{0}}^{*} & \mathbf{I}
\end{array}\right]\left[\begin{array}{l}
\mathbf{f}_{\mathbf{0}}^{-} \\
\mathbf{f}_{\mathbf{0}}^{+}
\end{array}\right]
$$


where $\mathbf{R}$ represents the real medium's reflection response at the transparent surface and $\mathbf{R}_{\mathbf{0}}$ the corresponding reflectivity operator in the reference (e.g., migration velocity) model. Again, the $*$ superscript denotes complex conjugation, that is, the reverse-time version of the corresponding operator. For the sake of notation, both systems of equations 5 can be written in a more compact form as $\mathbf{g}=\mathbf{M f}$, and $\mathbf{g}_{\mathbf{0}}=\mathbf{M}_{\mathbf{0}} \mathbf{f}_{\mathbf{0}}$, where $\mathbf{M}$ and $\mathbf{M}_{\mathbf{0}}$ are the real- and reference-medium Marchenko operators, respectively. Given the known background model, quantities $\mathbf{g}_{\mathbf{0}}$ and $\mathbf{R}_{\mathbf{0}}$ are calculated by forward modeling, so that one may have access to $\mathbf{f}_{\mathbf{0}}$ by inversion. Next, we introduce the perturbation quantities $\delta \mathbf{g}=\mathbf{g}-\mathbf{g}_{\mathbf{0}}$, and $\delta \mathbf{f}=\mathbf{f}-\mathbf{f}_{\mathbf{0}}$, which represent the scattered-wavefield effects in $\mathbf{g}$ and $\mathbf{f}$ as a result of the differences between the real and reference media. By subtracting the Marchenko system in the reference from that in the actual medium, an extended Marchenko system for scattered fields is derived,

$$
\left[\begin{array}{c}
-\delta \mathbf{g}^{-} \\
\delta \mathbf{g}^{+*}
\end{array}\right]=\left[\begin{array}{cc}
\mathbf{I} & -\mathbf{R} \\
-\mathbf{R}^{*} & \mathbf{I}
\end{array}\right]\left[\begin{array}{l}
\delta \mathbf{f}^{-} \\
\delta \mathbf{f}^{+}
\end{array}\right]+\left[\begin{array}{cc}
\mathbf{0} & -\delta \mathbf{R} \\
-\delta \mathbf{R}^{*} & \mathbf{0}
\end{array}\right]\left[\begin{array}{l}
\mathbf{f}_{\mathbf{0}}^{-} \\
\mathbf{f}_{\mathbf{0}}^{+}
\end{array}\right] .
$$

As can be noted, this new representation, $\delta \mathbf{g}=\mathbf{M} \delta \mathbf{f}+\delta \mathbf{M f}_{\mathbf{0}}$, describes the relationship between the unknown Green's function perturbation $\delta \mathbf{g}$, in terms of $\mathbf{R}, \mathbf{R}_{\mathbf{0}}$, and $\mathbf{f}_{\mathbf{0}}$, where one identifies the perturbation operator $\delta \mathbf{M}=\mathbf{M}-\mathbf{M}_{\mathbf{0}}$ as the fundamental element reflecting discrepancies between the two media. We referrer to such formulation as Scattering Marchenko, or S-Marchenko. In real applications, care must be taken into account when considering the proper scaling between the two reflection responses. Solving for $\delta \mathbf{f}^{+}$and $\delta \mathbf{f}^{-}$ is done by restricting the space-time support to the interval $-t_{d}\left(\mathbf{x}_{\mathbf{r}}, \mathbf{x}_{\mathbf{f}}\right) \leq t \leq t_{d}\left(\mathbf{x}_{\mathbf{r}}, \mathbf{x}_{\mathbf{f}}\right)$, where the windowing operator, $\boldsymbol{\Psi}$, separates causal from acausal events. This operator admits all events before the first arrival in $\delta \mathbf{g}^{ \pm}$while eliminates any other event mapping outside the window (Wapenaar et al., 2014b). Introducing this causality constraint allows 
us to recast equation 6 into a system that relates focusing-function perturbations $\delta \mathbf{f}$ only to the focusing functions in the background $\mathbf{f}_{\mathbf{0}}$, i.e.

$$
\left[\begin{array}{cc}
\mathbf{0} & \boldsymbol{\Psi} \delta \mathbf{R} \\
\mathbf{\Psi} \delta \mathbf{R}^{*} & \mathbf{0}
\end{array}\right]\left[\begin{array}{c}
\mathbf{f}_{\mathbf{0}}^{-} \\
\mathbf{f}_{\mathbf{0}}^{+}
\end{array}\right]=\left[\begin{array}{cc}
\mathbf{I} & -\boldsymbol{\Psi} \mathbf{R} \\
-\mathbf{\Psi} \mathbf{R}^{*} & \mathbf{I}
\end{array}\right]\left[\begin{array}{c}
\delta \mathbf{f}^{-} \\
\delta \mathbf{f}^{+}
\end{array}\right] .
$$

A simplified form of equation 7 reads $\delta \mathbf{M}^{\prime} \mathbf{f}_{\mathbf{0}}=\mathbf{M}^{\prime} \delta \mathbf{f}$, where $\mathbf{M}^{\prime}$ and $\delta \mathbf{M}^{\prime}$ take into account the influence of the windowing function $\boldsymbol{\Psi}$ acting on $\mathbf{R}$. Finally, the aforementioned equation is solved as a the constrained optimization problem,

$$
\min _{\delta \mathbf{f}}\left\|\mathbf{M}^{\prime} \delta \mathbf{f}-\delta \mathbf{M}^{\prime} \mathbf{f}_{\mathbf{0}}\right\|_{2}^{2} \quad \text { s.t. } \quad \mathbf{g}_{\mathbf{0}}=\mathbf{M}_{\mathbf{0}} \mathbf{f}_{\mathbf{0}}
$$

From a practical perspective, numerical implementation is done by following a two-step, alternated optimisation process. First, full-waveform forward modeling in the reference model is implemented to reconstruct $\mathbf{R}_{\mathbf{0}}$ and $\mathbf{g}_{\mathbf{0}}$, followed by inversion of the full (i.e., without windowing) Marchenko system in the reference model for an estimate of $\mathbf{f}_{\mathbf{0}}$. Next, the true-medium response, $\mathbf{R}$; together with, the modeled reference-model response, $\mathbf{R}_{\mathbf{0}}$, and $\mathbf{f}_{\mathbf{0}}$ from the previous step, are used to invert for $\delta \mathbf{f}$.

\section{BAND-LIMITED WAVEFIELD EXTRAPOLATION}

The idea of reproducing full wavefield responses from virtual sources at depth using focusing operators as presented in the previous section requires several key preprocessing steps that may introduce inaccuracies or even hinder the implementation of Marchenko redatuming in real data sets (Ravasi et al., 2016; da Costa Filho et al., 2017; Jia et al., 2018; Mildner et al., 2019a). The original approach demands knowledge of the acquisition wavelet and dense spatial distribution of sources and receivers. In effect, the surface reflection response needs to be accurately deconvolved by the source wavelet. As Mildner et al. (2019b) showed, 
an erroneous removal of the source fingerprint leads to artifacts in local images based on redatumed data. Additionally, the formalism presented above does not consider the effects of the free surface, as a result, surface-related multiples elimination (SRME, Verschuur et al., 1992; van Borselen et al., 1996; Amundsen, 2001) is a requirement. While most field data sets require 3D SRME for reasonable multiple suppression (Dragoset et al., 2010), it is also well-known that this process may introduce distortions to target signals, thus affecting data quality before redatuming. Alternatively, in the context of Marchenko redatuming, a natural way to circumvent the heavy toll of preprocessing consists in transforming the up- and downgoing Green's functions (equations 1 and 2), with a band-limited aerial source via multidimensional convolution (equation 9). Such a source can seamlessly account for the effects of all orders of internal and surface-related multiples enclosed in the downgoing vertical particle velocity measured at the receivers side (Ravasi, 2017).

\section{Rayleigh Marchenko}

Provided availability of dual-sensor data, one can use the recorded pressure $p\left(\mathbf{x}_{\mathbf{r}}, \mathbf{x}_{\mathbf{s}} ; t\right)$ to decompose the vertical particle velocity $v_{z}\left(\mathbf{x}_{\mathbf{r}}, \mathbf{x}_{\mathbf{s}} ; t\right)$ into its up- and downgoing constituents $v_{z}^{ \pm}\left(\mathbf{x}_{\mathbf{r}}, \mathbf{x}_{\mathbf{s}} ; t\right)$ (Wapenaar, 1998). In marine seismic data studies, and in particular for oceanbottom cable $(\mathrm{OBC})$ or node $(\mathrm{OBN})$ surveys, such fields are emitted from sources at a different level, $\mathbb{D}_{s}$, above the receivers line and below a free surface, see Figure 1. Ravasi (2017) used $v_{z}^{+}$as aerial source at the receivers level which then propagate into the medium with the help of the Green's functions, a process that grants access to the pressure field that would have been measured at $\mathbb{D}_{s}$ as if there was a source at the focal point, $\mathbf{x}_{\mathbf{f}}$. This convolutional operation is described by equation 9 . What is also interesting to note, is that $v_{z}^{+}\left(\mathbf{x}_{\mathbf{r}}, \mathbf{x}_{\mathbf{s}} ; t\right)$ and $v_{z}^{-}\left(\mathbf{x}_{\mathbf{r}}, \mathbf{x}_{\mathbf{s}} ; t\right)$ are related one another through the reflection response 
$R\left(\mathbf{x}_{\mathbf{r}}, \mathbf{x}_{\mathbf{r}}^{\prime}\right)$ at the receiver level, as indicated by the following Rayleigh integral representation (equation 10):

$$
\begin{aligned}
p^{ \pm}\left(\mathbf{x}_{\mathbf{f}}, \mathbf{x}_{\mathbf{s}}\right) & =\int_{\partial \mathbb{D}_{0}} v_{z}^{+}\left(\mathbf{x}_{\mathbf{r}}, \mathbf{x}_{\mathbf{s}}\right) g^{ \pm}\left(\mathbf{x}_{\mathbf{f}}, \mathbf{x}_{\mathbf{r}}\right) d \mathbf{x}_{\mathbf{r}} \\
-v_{z}^{-}\left(\mathbf{x}_{\mathbf{r}}^{\prime}, \mathbf{x}_{\mathbf{s}}\right) & =\int_{\partial \mathbb{D}_{0}} v_{z}^{+}\left(\mathbf{x}_{\mathbf{r}}, \mathbf{x}_{\mathbf{s}}\right) R\left(\mathbf{x}_{\mathbf{r}}, \mathbf{x}_{\mathbf{r}}^{\prime}\right) d \mathbf{x}_{\mathbf{r}}
\end{aligned}
$$

The advantage of considering vertical particle velocities is that they naturally contain the effects of surface-related multiples while preserving the band-limited character of observed seismic fields. Secondly, contrary to the data required by the Reflection operator (which demand accurate absolute-value scaling), $v_{z}^{ \pm}$fields are naturally scaled with respect to each other, therefore, estimation of an absolute scaling factor is not required in practical implementations (van der Neut et al., 2015c). Introducing equations 1 and 2 into 9 with the help of equation 10 eliminates the contribution of the reflection response $R\left(\mathbf{x}_{\mathbf{r}}, \mathbf{x}_{\mathbf{r}}^{\prime}\right)$ yielding,

$$
\begin{aligned}
-p^{-}\left(\mathbf{x}_{\mathbf{f}}, \mathbf{x}_{\mathbf{s}}\right) & =\int_{\partial \mathbb{D}_{\mathbf{0}}} v_{z}^{+}\left(\mathbf{x}_{\mathbf{r}}, \mathbf{x}_{\mathbf{s}}\right) f^{-}\left(\mathbf{x}_{\mathbf{r}}, \mathbf{x}_{\mathbf{f}}\right) d \mathbf{x}_{\mathbf{r}}+\int_{\partial \mathbb{D}_{\mathbf{0}}} v_{z}^{-}\left(\mathbf{x}_{\mathbf{r}}^{\prime}, \mathbf{x}_{\mathbf{s}}\right) f^{+}\left(\mathbf{x}_{\mathbf{r}}^{\prime}, \mathbf{x}_{\mathbf{f}}\right) d \mathbf{x}_{\mathbf{r}}^{\prime} \\
p^{+*}\left(\mathbf{x}_{\mathbf{f}}, \mathbf{x}_{\mathbf{S}}\right) & =\int_{\partial \mathbb{D}_{\mathbf{0}}} v_{z}^{-*}\left(\mathbf{x}_{\mathbf{r}}^{\prime}, \mathbf{x}_{\mathbf{S}}\right) f^{-}\left(\mathbf{x}_{\mathbf{r}}^{\prime}, \mathbf{x}_{\mathbf{f}}\right) d \mathbf{x}_{\mathbf{r}}^{\prime}+\int_{\partial \mathbb{D}_{\mathbf{0}}} v_{z}^{+*}\left(\mathbf{x}_{\mathbf{r}}, \mathbf{x}_{\mathbf{s}}\right) f^{+}\left(\mathbf{x}_{\mathbf{r}}, \mathbf{x}_{\mathbf{f}}\right) d \mathbf{x}_{\mathbf{r}}
\end{aligned}
$$

The set of equations 11 and 12 represent the Rayleigh-Marchenko formulation for singlesided redatuming problems (Ravasi, 2017), hereafter referred to as R-Marchenko. An alternative derivation is found directly from one-way reciprocity theorems of the convolution and correlation type with interaction quantity $\partial_{z}\left\{P_{A}^{+} V_{z, B}^{-}-P_{A}^{-} V_{z, B}^{+}\right\}$(Wapenaar and Grimbergen, 1996; Slob and Wapenaar, 2017). Referring back to the discrete formulation of equations 1 and 2, the R-Marchenko system can also be expressed in matrix-vector notation by defining $\mathbf{V}_{z}^{ \pm}$as multidimensional discrete filters applied to the focusing functions $\mathbf{f}^{ \pm}$to reconstruct, $\mathbf{p}^{ \pm}$, the up- and downgoing subsurface pressure wavefields at the focusing level 
$\partial \mathbb{D}_{i}$. Without loss of generality it is written as:

$$
\left[\begin{array}{c}
-\mathbf{p}^{-} \\
\mathbf{p}^{+*}
\end{array}\right]=\left[\begin{array}{cc}
\mathbf{V}_{z}^{+} & \mathbf{V}_{z}^{-} \\
\mathbf{V}_{z}^{-*} & \mathbf{V}_{z}^{+*}
\end{array}\right]\left[\begin{array}{l}
\mathbf{f}^{-} \\
\mathbf{f}^{+}
\end{array}\right]
$$

Solving the system of equations 13 relies on a causality argument along the lines of the one stated in the previous section. A new window $\Theta$ is built to remove all causal and acausal events within the time interval $-t_{d}\left(\mathbf{x}_{\mathbf{s}}, \mathbf{x}_{\mathbf{f}}\right) \leq t \leq t_{d}\left(\mathbf{x}_{\mathbf{s}}, \mathbf{x}_{\mathbf{f}}\right)$, i.e $\boldsymbol{\Theta} \mathbf{p}^{-}=\mathbf{0}$ and $\boldsymbol{\Theta} \mathbf{p}^{+}=\mathbf{0}$. Application of this muting function to both sides of equations 11 and 12 allows the focusing functions to be retrieved from the following system

$$
\left[\begin{array}{cc}
\mathbf{0} & -\boldsymbol{\Theta} \mathbf{V}_{z}^{-} \\
-\boldsymbol{\Theta} \mathbf{V}_{z}^{+*} & \mathbf{0}
\end{array}\right]\left[\begin{array}{c}
\mathbf{f}_{d}^{+} \\
\mathbf{f}_{d}^{+}
\end{array}\right]=\left[\begin{array}{cc}
\mathbf{\Theta} \mathbf{V}_{z}^{+} & \boldsymbol{\Theta} \mathbf{V}_{z}^{-} \\
\mathbf{\Theta} \mathbf{V}_{z}^{-*} & \boldsymbol{\Theta} \mathbf{V}_{z}^{+*}
\end{array}\right]\left[\begin{array}{c}
\mathbf{f}^{-} \\
\mathbf{f}_{m}^{+}
\end{array}\right]
$$

where $\mathbf{f}_{m}^{+}$and $\mathbf{f}^{-}$are evaluated in terms of an initial focusing function typically replaced by the direct part of a seismic response obtained from a given smooth model, $\mathbf{f}_{d}^{+} \approx \mathbf{g}_{d}^{*}$. The focusing process for both, Marchenko and R-Marchenko schemes, depends on the correct estimation of $\mathbf{g}_{d}^{*}$. Depending on overburden complexity in terms of fine-layer impedance variations, additional amplitude and phase corrections may be accounted for in a second focusing step as a way to compensate for the effects of short-period multiples (Dukalski et al., 2019).

The one-way focusing functions in equation 4 can be found by solving the coupled Marchenko equations either by Neumann-series iterative substitution (e.g Wapenaar et al., 2014b; van der Neut et al., 2015b), or direct inversion (iterative or otherwise, e.g van der Neut et al., 2015a; Ravasi, 2017). Note that equation 4 is of the form $\mathbf{y}=[\mathbf{I}-\mathbf{A}] \mathbf{x}$, a Fredholm integral equation of the second kind, which is amenable to solutions in terms of Neumann series expansion. A detailed analysis of this kind of solutions is offered by Dukalski 
and de Vos (2017). It is shown that for strong scattering regimes, and in the presence of surface-related multiples, convergence is not necessarily guaranteed. More broadly speaking, the convergence of such series depends on whether or not the spectral radius of the operator A is bounded by unity. Unlike the standard Marchenko equations, the Rayleigh Marchenko system is a Fredholm integral of the first kind, in consequence, it cannot be expanded into a Neumann series. Without additional constraints or conditions, it is not possible to solve the R-Marchenko for the focusing functions using iterative substitution, thus direct inversion must be employed instead. Either equations 4 or 14 can be treated as a linear inverse problem (van der Neut et al., 2015a) where least-squares iterative solvers (e.g., LSQR - Paige and Saunders (1982)) prove to converge to a solution whose residuals decay monotonically, making such solvers an attractive option for Marchenko problems. Furthermore, an advantage of using direct inversion lies in the fact that data does not necessarily need to be complete. Indeed, even in cases where it is irregularly sampled or polluted with noise, one may impose constraints in such a way that a satisfactory solution can still be estimated (Haindl et al., 2021). In such cases, experience has shown that sparsity-promoting inversion (van den Berg and Friedlander, 2009; Hennenfent and Herrmann, 2008; Beck and Teboulle, 2009) may be even more suitable than least-squares inversion - provided that a suitable sparsifying basis transformation is known and available (Haindl et al., 2021).

\section{Scattering Rayleigh Marchenko}

Building on the ideas of Vasconcelos and Sripanich (2019), we present an extended formulation of the previously described R-Marchenko method. This framework aims at reconstructing perturbed focusing functions with respect to a background medium from band-limited data while preserving the effects of the surface-related multiples while also allowing for substantial 
flexibility in terms of acquisition geometries. As a result, corresponding scattered pressure fields at depth are retrieved. Even though the R-Marchenko scheme relaxes some of the acquisition and preprocessing requirements, it still suffers from similar difficulties traditional Marchenko endures when it comes to wavefield extrapolation in complex media. In subsurface characterized by geologic intrusions exhibiting high impedance contrast, as is the case of diapiric traps. In sub-salt, or sub-basalt environments, even a fairly accurate estimation of a direct wave as a proxy for the initial focusing function in Marchenko may not be sufficient to guarantee its success. Once again, we rely on the availability of high-end migration models containing information on the salt's sharp structure and contrast magnitudes to define two independent Rayleigh Marchenko integral systems in the matrix-vector form (equation 13), one describing the relationship between focusing and pressure fields in the true medium and the other is a reference (e.g., migration) model,

$$
\left[\begin{array}{c}
-\mathbf{p}^{-} \\
\mathbf{p}^{+*}
\end{array}\right]=\left[\begin{array}{cc}
\mathbf{V}_{z}^{+} & \mathbf{V}_{z}^{-} \\
\mathbf{V}_{z}^{-*} & \mathbf{V}_{z}^{+*}
\end{array}\right]\left[\begin{array}{l}
\mathbf{f}^{-} \\
\mathbf{f}^{+}
\end{array}\right] \quad, \quad\left[\begin{array}{c}
-\mathbf{p}_{\mathbf{0}}^{-} \\
\mathbf{p}_{\mathbf{0}}^{+*}
\end{array}\right]=\left[\begin{array}{cc}
\mathbf{V}_{0, z}^{+} & \mathbf{V}_{0, z}^{-} \\
\mathbf{V}_{0, z}^{-*} & \mathbf{V}_{0, z}^{+*}
\end{array}\right]\left[\begin{array}{l}
\mathbf{f}_{\mathbf{0}}^{-} \\
\mathbf{f}_{\mathbf{0}}^{+}
\end{array}\right] .
$$

Here, up/down-going pressure fields $\mathbf{p}^{ \pm}$are given in terms of focusing functions $\mathbf{f}^{ \pm}$ through the real medium's vertical particle velocity measured at the surface $\mathbf{V}_{z}^{ \pm}$. Similarly, in the background model, $\mathbf{V}_{0, z}^{ \pm}$relates focusing functions $\mathbf{f}_{0}^{ \pm}$with pressure fields $\mathbf{p}_{0}^{ \pm}$. For the sake of notation, both systems in equations 15 can be written in a more compact form as $\mathbf{p}=\mathbf{V f}$, and $\mathbf{p}_{\mathbf{0}}=\mathbf{V}_{\mathbf{0}} \mathbf{f}_{\mathbf{0}}$, respectively. Since we assume the background model to be known, quantities $\mathbf{p}_{\mathbf{0}}^{ \pm}$and $\mathbf{V}_{\mathbf{0}, \mathbf{z}}^{ \pm}$can be estimated by forward modeling, as a result one may have access to $\mathbf{f}_{\mathbf{0}}$ by inversion of the reference system of equations 15 , or 5 when the broadband fields $\mathbf{g}_{\mathbf{0}}^{ \pm}$and $\mathbf{R}_{\mathbf{0}}$ are modeled instead. Next, we introduce the perturbation quantities $\delta \mathbf{p}=\mathbf{p}-\mathbf{p}_{\mathbf{0}}$, and $\delta \mathbf{f}=\mathbf{f}-\mathbf{f}_{\mathbf{0}}$. They represent the scattered-wavefield effects in $\mathbf{p}$ and $\mathbf{f}$ as a result of the differences between the real and reference media. In the light of this definition, 
any feature in the data that can not be correctly explained by the migration model should appear in the perturbations. In terms of focusing functions and redatuming operators, the perturbed pressure fields arise after combining the Marchenko system in the actual medium, $\mathbf{p}=\mathbf{V f}$, with the one in the reference, $\mathbf{p}_{\mathbf{0}}=\mathbf{V}_{\mathbf{0}} \mathbf{f}_{\mathbf{0}}$. Therefore, an alternative Marchenko system for scattered fields reads as follows,

$$
\left[\begin{array}{c}
-\delta \mathbf{p}^{-} \\
\delta \mathbf{p}^{+*}
\end{array}\right]=\left[\begin{array}{cc}
\mathbf{V}_{z}^{+} & \mathbf{V}_{z}^{-} \\
\mathbf{V}_{z}^{-*} & \mathbf{V}_{z}^{+*}
\end{array}\right]\left[\begin{array}{l}
\delta \mathbf{f}^{-} \\
\delta \mathbf{f}^{+}
\end{array}\right]+\left[\begin{array}{cc}
\delta \mathbf{V}_{z}^{+} & \delta \mathbf{V}_{z}^{-} \\
\delta \mathbf{V}_{z}^{-*} & \delta \mathbf{V}_{z}^{+*}
\end{array}\right]\left[\begin{array}{l}
\mathbf{f}_{\mathbf{0}}^{-} \\
\mathbf{f}_{\mathbf{0}}^{+}
\end{array}\right]
$$

with $\delta \mathbf{p}=\mathbf{V} \delta \mathbf{f}+\delta \mathbf{V} \mathbf{f}_{\mathbf{0}}$. We refer to this new representation as Scattering-RayleighMarchenko, or as SR-Marchenko. It describes the relationship between the unknown pressure field perturbations $\delta \mathbf{p}$, in terms of $\mathbf{f}_{\mathbf{0}}$ and those in $\delta \mathbf{f}$ via vertical particle velocity propagator V. A key element of our system in equation 16 is the the perturbation operator $\delta \mathbf{V}=\mathbf{V}-\mathbf{V}_{\mathbf{0}}$ which captures the differences between data from the reference model and field data, i.e., the true medium. When it comes to applications to standard surveys, it is important that the data in the operators $\mathbf{V}$ and $\mathbf{V}_{\mathbf{0}}$ are properly scaled relative to one another. We ensure operator compatibility by transplanting the measured down-going vertical particle velocity, $v_{z}^{+}$, into the reference medium at the receiver level $\mathbf{V}_{z}^{+} \rightarrow \mathbf{V}_{0, z}^{+}$, and used it as aerial source. The seismic response of the reference model is then given by the one-way Rayleigh integral representation (equation 10) through a numerically modeled reflection response $\mathbf{R}_{\mathbf{0}}$. Then, the following conditions relate the wavefield propagation in the true and reference medium: $\mathbf{V}_{0, z}^{-}=-\mathbf{R}_{\mathbf{0}} \mathbf{V}_{z}^{+}, \mathbf{V}_{0, z}^{+}=\mathbf{V}_{z}^{+}$. The fields in the reflection response $\mathbf{R}_{\mathbf{0}}$ do not consider multiples related to reflectors above the level $\partial \mathbb{D}_{0}$, while introducing additional information from the background model into the up-going vertical particle velocity $v_{0, z}^{-}$. This argument in the SR-Marchenko system leads to a further simplification of the perturbation operator, as $\delta \mathbf{V}_{z}^{+}=\mathbf{0}$ and $\delta \mathbf{V}_{z}^{-}=\mathbf{V}_{z}^{-}+\mathbf{R}_{0} \mathbf{V}_{z}^{+}$since all medium perturbations occur below $\partial \mathbb{D}_{0}$, so 
field perturbations only pertain to the upgoing fields.

Taking into consideration that the system of equations 16 is underdetermined, additional restrictions need to be imposed in order to reduce the number of unknowns. Once more, we invoke the same causality arguments introduced in the previous section and apply a muting window to reformulate equation 16 into a system that only relates focusing functions in the background, $\mathbf{f}_{\mathbf{0}}$, with their perturbations counterparts, $\delta \mathbf{f}$. i.e.

$$
\left[\begin{array}{cc}
\mathbf{0} & -\boldsymbol{\Theta} \delta \mathbf{V}_{z}^{-} \\
-\boldsymbol{\Theta} \delta \mathbf{V}_{z}^{-*} & \mathbf{0}
\end{array}\right]\left[\begin{array}{l}
\mathbf{f}_{\mathbf{0}}^{-} \\
\mathbf{f}_{\mathbf{0}}^{+}
\end{array}\right]=\left[\begin{array}{cc}
\mathbf{\Theta} \mathbf{V}_{z}^{+} & \boldsymbol{\Theta} \mathbf{V}_{z}^{-} \\
\mathbf{\Theta} \mathbf{V}_{z}^{-*} & \boldsymbol{\Theta} \mathbf{V}_{z}^{+*}
\end{array}\right]\left[\begin{array}{l}
\delta \mathbf{f}^{-} \\
\delta \mathbf{f}^{+}
\end{array}\right],
$$

which for simplicity, it can also be expressed in compact format as $\delta \mathbf{V}^{\prime} \mathbf{f}_{\mathbf{0}}=\mathbf{V}^{\prime} \delta \mathbf{f}$, with $\mathbf{V}^{\prime}$ and $\delta \mathbf{V}^{\prime}$ encoding the influence of the windowing function $\boldsymbol{\Theta}$ acting on $\mathbf{V}$ and $\delta \mathbf{V}$. Unlike equation 4, equation 17 is a Fredholm integral of the first kind and does not admit a solution in terms of iterative substitution (van der Neut et al., 2015b). Finally, similarly to equation 7, the aforementioned equation is solved as a two-step optimization problem:

$$
\min _{\delta \mathbf{f}}\left\|\mathbf{V}^{\prime} \delta \mathbf{f}-\delta \mathbf{V}^{\prime} \mathbf{f}_{\mathbf{0}}\right\|_{2}^{2} \quad \text { s.t. } \quad \mathbf{g}_{\mathbf{0}}=\mathbf{M}_{\mathbf{0}} \mathbf{f}_{\mathbf{0}}
$$

From an operational point of view, numerical implementation is done first by constructing $\mathbf{R}_{\mathbf{0}}$ and $\mathbf{g}_{\mathbf{0}}$ using full-waveform forward modelling in the reference model, followed by inversion of the reference Marchenko system in equations 5 to find $\mathbf{f}_{\mathbf{0}}$. Second, we solve the system of equations 17 for $\delta \mathbf{f}$ using the true-medium responses, $\mathbf{V}_{z}^{ \pm}$, the perturbation operators $\delta \mathbf{V}_{z}^{ \pm}$, and $\mathbf{f}_{\mathbf{0}}$ from the previous step. Finally, the perturbation in the pressure field $\delta \mathbf{p}$ is given according to equation 16 and the total redatumed field is then found as $\mathbf{p}=\mathbf{p}_{\mathbf{0}}+\delta \mathbf{p}$. 


\section{SUB-SALT SYNTHETIC EXAMPLES}

We illustrate the practical implementation of our scattering-based redatuming schemes, S-Marchenko and SR-Marchenko, and compare their performance against conventional Marchenko and R-Marchenko, respectively. With the idea of evaluating the effectiveness of these methods, we designed a complex model describing different orders of scattering, which exhibits a high impedance contrast overburden mimicking the effects of an inhomogeneous (dirty) salt body (Figure 2). Beneath the complex overburden, this model is characterized by laterally heterogeneous sediments, interbed discontinuities, and sharp unconformities. The medium expands $16.26 \mathrm{~km}$ in the horizontal direction and extends up to $8.0 \mathrm{~km}$ in depth. In addition to that, the reference model we use as a supplement in the following tests resembles the output of conventional high-end velocity model building, where the salt and sea bottom are represented by sharp discontinuities, while sediment wave speed is kept smooth and salt wavespeed is constant. We first demonstrate wavefield reconstruction at virtual receivers located on a target level inside the medium, followed by interferometric redatuming. The former is carried through multidimensional deconvolution, implemented to build local extended images. Finally, we migrate the extended images and discuss the main consequences of using accurate redatumed full-wavefields accounting for internal and free multiple reflections.

\section{Wavefield extrapolation beneath complex overburden}

The acquisition set up of our numerical experiments consists of 201 receivers regularly distributed every $40 \mathrm{~m}$. To simulate the full reflection response $\mathbf{R}$ in the true medium, we use a finite-difference acoustic solver and sequentially inject 201 sources on the model surface. 
It is worth mentioning that the shot gathers are recorded for $8 \mathrm{~s}$, allowing the observation of deeper events and that their maximum aperture is $4 \mathrm{~km}$. Similarly, a numerically modeled reflection response, $\mathbf{R}_{0}$, in the migration velocity model is implemented. For data modeling, a broadband impulse-source with a flat spectrum in the range of 1 to $80 \mathrm{~Hz}$ is used. It should be noted that the relative source amplitude is preserved to ensure survey compatibility when constructing the perturbation operator $\delta \mathbf{R}$. The extrapolation algorithm starts by modeling the full transmission response in the background model associated with a focal point at $4.4 \mathrm{~km}$ depth in the middle of the physical domain at $8.13 \mathrm{~km}$ using a $20 \mathrm{~Hz}$ Ricker wavelet (Figure 3d). For the original Marchenko redatuming scheme, the coda part of this transmission is typically discarded and only the direct part is preserved to find the initial focusing function, $\mathbf{f}_{\mathbf{d}}^{+}$, either by inversion of $\mathbf{i}=\mathbf{g}_{\mathbf{0}, \mathbf{d}} \mathbf{f}_{\mathbf{d}}^{+}$, or approximated by time-reversal $\mathbf{f}_{\mathbf{d}}^{+} \approx \mathbf{g}_{\mathbf{0}, \mathbf{d}}^{*}$. Contrary to that strategy, in S-Marchenko, we retain the full wavefield to solve for the true focusing function, $\mathbf{f}_{\mathbf{0}}$ (Figure $3 \mathrm{a}$ ), in this model and we show that the coda part of $\mathbf{g}_{\mathbf{0}}$ carries essential information for redatuming. Since no windowing constrains intervene in this step, internal multiples arriving after the direct wave contribute to the focusing process, as suggested by events appearing outside of the domain that would have been muted otherwise by a windowing operator. Now that $\mathbf{f}_{\mathbf{0}}$ is available (Figure 3a), focusing functions perturbations are estimated by our second inversion step. Figure $3 \mathrm{~b}$ shows that $\partial \mathbf{f}$ contains additional events explained by the reflection data in the true medium that add further information to the total focusing function, $\mathbf{f}$ in Figure 3c. As observed in Figure 3e, the scattered Green's function $\delta \mathbf{g}$ retrieves reflection events coming from the deeper parts of the medium and internal multiples arriving after $3.5 \mathrm{~s}$ that do not appear in the reference Green's function ( $\mathbf{g}_{\mathbf{0}}$ in Figure 3d). Comparing the information in the S-Marchenko based focusing function (Figure 4a) to that in the standard approach (Figure 4b) exposes events 
mapping outside the range $-t_{d}\left(\mathbf{x}_{\mathbf{r}}, \mathbf{x}_{\mathbf{f}}\right) \leq t \leq t_{d}\left(\mathbf{x}_{\mathbf{r}}, \mathbf{x}_{\mathbf{f}}\right)$ violating the causality argument invoked in defining the windowing operator employed in Marchenko schemes. In practice, our S-Marchenko scheme can only update $\delta \mathbf{f}$ within the $-t_{d}\left(\mathbf{x}_{\mathbf{r}}, \mathbf{x}_{\mathbf{f}}\right) \leq t \leq t_{d}\left(\mathbf{x}_{\mathbf{r}}, \mathbf{x}_{\mathbf{f}}\right)$ interval, but both the $\delta \mathbf{f}$ update and the full-time range of $\mathbf{f}_{\mathbf{0}}$ contribute to enhanced retrieval of $\delta \mathbf{g}$ - we provide further thoughts on this in the Discussion section. Furthermore, Figure 4c displays a substantial trace mismatch among focusing operators. At this point, we verify retrieved Green's function accuracy by comparing it with a directly modeled benchmark (Figure 5a). The recovered responses, corresponding to the same selected focal point, are depicted in Figure 5b for the S-Marchenko, and Figure 5c for the standard Marchenko. While to some extent the fields retrieved by the conventional scheme in Figure 5c match those in Figure 5a, upon closer inspection we observe substantial amplitude inaccuracies and many missing events. Likewise, examining the waveforms of the traces extracted from a receiver located at $8.6 \mathrm{~km}$ reveals a mismatch even for the early reflections. Such behavior is more pronounced for events arriving at later times (Figure 5d). On the other hand, our scattering-based equivalent (Figure 5b) achieves a much better fit especially for the earlier arrivals, not only the amplitudes but also the kinematics of the whole gather is substantially better retrieved. In this case, trace evaluation against the benchmark (Figure 5d) supports the hypothesis that including more accurate transmitted wavefields (implicit in our $\mathbf{f}_{\mathbf{0}}$ fields), by means of a priori model information, can be critical for waveform fidelity in Marchenko-redatumed fields for media with this level of complexity.

Having exemplified the performance of Marchenko versus S-Marchenko, we move to our second experiment. In this case, the efficiency of R-Marchenko and SR-Marchenko is subject to evaluation. Note that opposite to the previous case study, we anticipate those Rayleigh-Marchenko versions could be more suitable candidates for real seismic surveys. 
Considering they require fewer preprocessing steps while still handling band-limited data containing both internal and free-surface multiples (Ravasi, 2017). In addition, the RMarchenko schemes can accommodate multiple acquisition geometries, as well as surveys with blended/simultaneous sources. To test these schemes, we extend our model with a 200 m water column to mimic an ocean bottom survey geometry. On the source side, pressure and vertical particle velocity data for 201 sources located at a $10 \mathrm{~m}$ depth are generated using a finite-difference vector-acoustic solver. Our synthetic ocean-bottom setup consists of 201 receivers regularly distributed every $40 \mathrm{~m}$ over a line at $200 \mathrm{~m}$ depth (Figure 2). Data are then decomposed into their up- and downgoing components (Wapenaar, 1998) followed by convolution with a $10 \mathrm{~Hz}$ Ricker wavelet to impose the band-limited character of the data to be used in the Rayleigh Marchenko operator $\mathbf{V}_{z}^{ \pm}$. Intentionally, this survey configuration allows us to use the full reflection response $\mathbf{R}_{0}$ from the previous experiment to generate the upgoing vertical particle velocity in the reference model, $\mathbf{V}_{0, z}^{-}$, through multidimensional convolution with its downgoing counterpart assumed to be observed in the true medium, $\mathbf{V}_{0, z}^{+}$. Because the medium is assumed to be unknown only below the receiver level (i.e., the sea bottom), using $\mathbf{V}_{z}^{+} \rightarrow \mathbf{V}_{0, z}^{+}$ensures proper operator relative scaling while at the same time simplifies the SR-Marchenko operator. In this paper, we purposefully include only results without free-surface effects in our Rayleigh-Marchenko analysis, because those effects are far more dominant then those related to internal multiples and salt-body interactions, while the overall behavior of the R- and SR-Marchenko methods shows analogous patterns as those seen in the examples below - they are representative of these two methods with or without free-surface effects. For a detailed comparison of R-Marchenko versus the original scheme in the presence of free-surface multiples, we refer the reader to Ravasi (2017). 
For redatuming purposes, we select a focal point at position $\mathbf{x}_{\mathbf{f}}=(4.4,6.0) \mathrm{km}$ and compute $\mathbf{g}_{\mathbf{0}}$ similar to the previous experiment. Figure $6 \mathrm{a}-6 \mathrm{c}$ depicts focusing functions from our band-limited ocean-bottom experiment. As in our previous study, the pressure response from the reference model (Figure 6d) offers access to crucial waveform information that otherwise underestimated or neglected. As above, the scattered pressure wavefield (Figure 6e) reveals waveform features that play a major role in the substantial improvement of the total retrieved pressure field (Figure 6e). In the interest of assessing the relative accuracy in both redatuming scenarios, we analyze the full focusing functions (Figure 7) since they largely influence redatuming performance. While in the SR-Marchenko a full-waveform transmitted field is encoded in the $\mathbf{f}_{\mathbf{0}}$ used to retrieve $\mathbf{f}$ (Figure $7 \mathrm{~b}$ ), in the original R-Marchenko, an initial focusing estimate extracted from the directly transmitted first-arrival in the reference medium is used instead. When comparing the focusing response from the proposed scattering-based method (Figure 7a) with the standard Rayleigh-based focusing operator (Figure 7b), we observe significant waveform discrepancies between one another. First, the focusing function in SR-Marchenko expands beyond limits in the muting window due to the more complex $\mathbf{f}_{\mathbf{0}}$, whereas the one in R-Marchenko lies within the domain imposed by causality. Second, events missing in Figure 7b appear in Figure 7a. Such arrivals are fundamental to ensure wavefield reconstruction and cancellation during the focusing process for retrieving $\mathbf{p}$ - ultimately resulting in better-quality retrieved pressure fields. Keeping in mind the introduced windowing constraints in our current SR-Marchenko scheme, the information outside the windowing function is inherited from the solution of $\mathbf{f}_{\mathbf{0}}$ in the reference model - but this information plays a role in the improved retrieval of events in $\delta$ f. A final remark can be made when comparing absolute and relative amplitudes of events retrieved by both methods (Figure 7c). There, we observe discrepancies between the two 
approaches that are noticeably significant - these differences are responsible for the absolute and offset-dependent amplitude differences between common events present in the $\mathbf{p}$ fields resulting from either approach.

Finally, we take a closer look at the recovered pressure fields and compare the performance of our SR-Marchenko approach relative to the R-Marchenko scheme. In Figure 8, pressure fields retrieved by both the R- and SR-Marchenko schemes are compared with that obtained by forward finite-difference modeling. A general inspection of Figure 8b and Figure 8c leads to the conclusion that SR-Marchenko consistently provides a robust estimation of the pressure fields considerably closer to the benchmark. Not only it is kinematically more accurate but also the amplitude is better resolved on most of the retrieved events, contrary to those from R-Marchenko. Furthermore, a close examination of the retrieved fields reveals events which are missing in Figure 8c that are recovered in SR-Marchenko. Likewise, pronounced artifacts observed in Figure 8c appear suppressed in Figure 8b. Following from our analysis of the focusing fields above, we attribute such improvement to the relevant refinements added by the perturbed pressure wavefields to the background-related ones, which subsequently enhances the final pressure response. Extracting a seismic trace interval for detailed comparison (Figure 8d) indicates an overall better waveform fit by the SR-Marchenko in contrast with the considerable misalignment in the R-Marchenko reconstruction.

\section{Target-oriented structural imaging}

In the next examples, we design a virtual survey, using the different flavors of Marchenkobased extrapolated wavefields, to recreate the data that would have been recorded at a datum level by an actual seismic experiment, as if sources and receivers were located just 
above the datum. Such data sets, in connection with traditional imaging conditions, are referred to as extended images (EIs, Vasconcelos et al., 2010; Vasconcelos and Rickett, 2013; Ravasi et al., 2014). From that perspective, the media below the target level back-scatters an upgoing pressure field $\mathbf{p}^{-}$when illuminated from above with an incident downgoing pressure field $\mathbf{P}^{+}$. This interaction is described by the convolutional process $\mathbf{p}^{-}=\mathbf{P}^{+} \mathbf{R}^{\cup}$, where $\mathbf{R}^{\cup}$ is the local reflection response at the focusing depth and assumes a reflection-free medium above that level. An estimation of the local reflection response, with illumination from above the target, is obtained by implementing multidimensional deconvolution, MDD (Wapenaar et al., 2008, 2011; Vasconcelos and Rickett, 2013); in Appendix A we describe in more detail our approach to pre-conditioned, time-domain MDD.

We start by reconstructing up- and downgoing pressure fields - by means of our Marchenko schemes - from the surface to an array of 151 virtual points spanning $3 \mathrm{~km}$ from 6.0 to 9.0 $\mathrm{km}$ horizontally, at a depth of $4.4 \mathrm{~km}$. The virtual survey is shown in Figure 2 enclosed by the dashed target box beneath the salt body. Figure 9 shows local reflection responses corresponding to a virtual source located at position $\mathbf{x}_{\mathbf{f}}=(4.4,7.5) \mathrm{km}$ where different extrapolated wavefields are used in the deconvolution process. Figure $9 \mathrm{~b}$ and $9 \mathrm{c}$ correspond to the virtual shot gathers resulting from employing broadband S-Marchenko and Marchenko based redatumed fields, respectively. We identify multiple coherent reflection events when evaluating against a synthetic gather modeled by finite-differences, using only the targetmedium properties (Figure 9a). Though here we display only one of the 151 common-source EIs retrieved by MDD, the behaviour displayed in these gathers is representative of the overall MDD results. In spite of the implicit numerical instabilities introduced by the MDD problem, a considerably more consistent reconstruction is achieved when relying on scattering-based Marchenko fields contrary to those from the original scheme. While some 
events are underestimated in the conventional method, Figure 9c, a stronger contribution for some of the events arriving after $1.5 \mathrm{~s}$ is appreciated in Figure 9b. Similarly, we present extended images obtained from band-limited redatumed data (Figure 9e and 9f). Contrary to the previous case, redatuming is done with the R-Marchenko and SR-Marchenko methods as input for the MDD. Figure 9e shows the result for SR-Marchenko whereas Figure 9f that for R-Marchenko. In these EI gathers, given the relatively simple geology beneath the salt, we do not expect to have strong interfering or discontinuous events. However, as observed in Figure 9e, the scattering-based method presents a more stable result than that of the conventional R-Marchenko when compared with the benchmark (Figure 9d). The prominent event discontinuities seen in both the R-Marchenko and original-scheme EIs result from poorly reconstructed events in their corresponding down-going fields - these do not capture the correct waveform behaviour as a function of subsurface offset in subsalt areas, leading to the illumination gaps in the retrieved EIs. S- and SR-Marchenko down-going fields, on the other hand, contain more arrivals with improved waveform fidelity, resulting in EI gathers with better subsurface-offset amplitude compensation that are considerably closer to the benchmark responses.

To assess how the differences in EI gathers may translate into imaging, we conduct target-oriented reverse time migration on the virtual shot gathers predicted by the different extrapolation methods. We compute 151 single-shot images and then stack them together to find the target-medium (box in Figure 2) migrated images Figure 10a-10c. This is inline with targeted Marchenko imaging approaches presented in previous studies (e.g., Wapenaar et al., 2014b; Ravasi, 2017). In this paper, for the sake of brevity, we refrain from showing comparisons between the Marchenko-based target-oriented images and surface-data-based migrated images of the target area. Overall, all of our Marchenko-based target images are 
superior to their surface-based RTM counterparts, with improvements comparable to those seen in previous studies (e.g., Wapenaar et al., 2014b; Ravasi, 2017), and with our S- and SR-Marchenko-based images being considerably better than those from surface-data-based RTM.

Here, we focus our analysis on comparing the imaging performance of our approaches versus existing Marchenko schemes, strictly in the context of target-oriented imaging. As such, Figure 10c corresponds to the conventional Marchenko local reflection response (Figure 9c), Figure 10b to the S-Marchenko result (Figure 9b), and Figure 10a to the RTM of the targetmedium benchmark Figure 9a. Our results show that the scattering-based image (Figure 10b) yields better fidelity to the benchmark in contrast with the traditional Marchenkobased image. In this case, the gain in resolution due to the better handling of complex salt-influenced wavefield is noticeable, especially for the large offsets as observed toward the image edges. Additionally, the details of stratigraphic features are also better resolved. As for the Marchenko-based image, the overall incorrectly extrapolated up and downgoing wavefields may result in structures that appear to be shallower than the true interfaces. This likely results from the improper handling of the direct-arrival phase distortions due to the salt body interactions. Regarding band-limited structural images corresponding to the Rayleigh-based Marchenko fields (Figure 10a-10c), a reliable reconstruction of the main reflectors can be observed in the SR-Marchenko image (Figure 10b). Unlike that of R-Marchenko (Figure 10c), where additional artifacts spotted for the deeper areas at about $7.0 \mathrm{~km}$, the SR-based image exhibits a closer representation of the subsurface as in the reconstruction in Figure 10a. Moreover, substantial differences in image quality are visible in the near surface (around $5.4 \mathrm{~km}$ ) as a consequence of an erroneous phase and amplitude estimation of the early arrivals in the redatumed wavefields (Figure 8d). 
For a more detailed analysis, we select multiple close-ups (indicated by the boxes in Figures 10 and 11) at different locations (Figures 12 and 13). For the first row in Figure 12 matching the green box in Figure 10, the most prominent differences between the SMarchenko and Marchenko RTM images are indeed in the shallow section of the target medium; still, noticeable differences between the two images exist also at deeper locations. In box 1 (top row), we see the the SR-Marchenko image yields a consistently more reliable representation of the complex seismic facies present in the benchmark image, which could impact potential interpretations both in an exploration or reservoir characterisation context. The next row corresponds to zoomed sections associated with box 2 across the multiple images. Even though most reflectors are present in both cases, a better continuity in the S-Marchenko image (Figure 12b) exposes a remarkable enhancement. We note that this is not the case for the reflectors reconstructed by Marchenko-based image, which in fact, displays a prominent image artefact in the left-center portion of Figure 12c. An additional observation concerns image reconstruction at far offsets: the last row in Figure 12, box 3 in Figure 10, displays a much better resolved reflector below $7.0 \mathrm{~km}$ for the S-Marchenko case, not only due to the accurate prediction of discontinuities but also because it can erroneously be interpreted as a mild dipping layer in the traditional Marchenko image. Figure 13 is the band-limited counterpart of Figure 12 for the same zoomed areas using the SR-Marchenko (Figure 13b) and R-Marchenko (Figure 13c) schemes contrasted against a $20 \mathrm{~Hz}$ RTM benchmark (Figure 13a). In general terms, similar improvements to the ones described for the S-Marchenko case are observed. Based on the previous examples, we expect that in the case of data containing surface-related multiples (SRM), SR- and R-Marchenko images would result in images like those in Figures 12 and 13, while conventional Marchenko imaging becomes significantly degraded by the presence of free-surface effects (Ravasi, 2017). 


\section{DISCUSSION}

In this paper, we present two novel redatuming scattering-based schemes for wavefield redatuming with the Marchenko framework whose objective is to account for highly complex overburden media such as subsalt or sub-basalt. With numerical experiments in a complex salt model, not only do we expose issues with the previous schemes, but we also convincingly show that our scattering approaches result in fields and images with considerably superior waveform fidelity. At the point of writing this study, these are the best scattering-based estimates we have to offer, however, we point out that they are approximate and likely could further improve. One key reason for this is our use of the windowing operator in the scattering schemes. As it stands, the windowing is a necessary constraint that allows for the elimination of Green's fuction perturbations from the Marchenko representations, e.g., as in equations 6 and 16, leading to the focusing-only systems that can numerically be solved. In practice, however, we indeed observe that the reference focusing functions $\mathbf{f}_{\mathbf{0}}$ contain events not only after the "direct arrival" - as is assumed by conventional Marchenko schemes but also before it, as seen in Figures 3 and 6 . This, in fact, is physically expected because in such complex media waveform triplications/caustics are common, so not only there are multiple events corresponding to the "direct" wave, but also other waves may arrive before some of the later paths of the ballistic/direct field. Here, we note that previous iterations of the Marchenko scheme (Wapenaar et al., 2014a) are shown to handle triplications in the retrieved coda, while those in the direct wave need to be properly included in the estimated initial direct wave. In our case: (1) caustics are handled on the direct/ballistic arrivals, (2) including multiple arrivals of the direct field in $\mathbf{f}_{0}$, with $(3)$ the correct dynamic/amplitude behaviour properly accounted for, because $\mathbf{f}_{0}$ is obtained by inversion. As a consequence of this, the behaviour we see in $\mathbf{f}_{\mathbf{0}}$ should, in principle, also apply to $\mathbf{f}$ and thus to $\delta \mathbf{f}$. However, 
because we rely on the same windowing operator - and thus the same causality arguments of conventional Marchenko schemes, our practical approach only retrieves updates to $\delta \mathbf{f}$ that lie within the causality window. Any waves contributing to $\delta \mathbf{f}$ that would lie outside the causality window are currently not retrieved by our practical schemes. In tests we conducted so far, even without estimating $\delta \mathbf{f}$ contributions lying outside the causality window, we obtain redatuming results with considerably better accuracy than those produced by the conventional approach. But that is not to say that these missing contributions do not matter, because there are still inaccuracies and missing events in our reconstructions (e.g., as seen in Figures 5 and 8). Building a better understanding of the role of focusing function wave components outside of the causality window, together with developing approaches that can estimate them, is the subject of ongoing research.

Continuing with the discussion on causality arguments and windowing, we point out that there is also an important distinction between the Reflection-operator- and Rayleigh-based Marchenko constructs. When defining causality constraints for the representations such as in equations 3 and 6 , the windowing operator has two purposes: the first is to annihilate $\mathbf{g}$ from the system, and the second is to isolate $\mathbf{f}_{m}^{+}$and $\mathbf{f}^{-}$. Mathematically, this is so because the representations have the form, e.g., $\mathbf{g}^{-}-\mathbf{f}^{-}=\mathbf{R} \mathbf{f}^{+}$, where the left-hand side contains a superposition of Green's and focusing functions. This is not the case for Rayleigh-Marchenko representations. There, the representations have the form, e.g., $-\mathbf{p}^{-}=\mathbf{V}_{z}^{-} \mathbf{f}^{+}+\mathbf{V}_{z}^{+} \mathbf{f}^{-}$, where there is no superposition of pressure fields and focusing functions on the left-hand side. As a result, the only role of causality constraints in Rayleigh-Marchenko is to eliminate the $\mathbf{p}$ wavefields from the system. This seemingly innocent difference between the two types of representations implies that windowing operators can in fact be different in practical implementations of either scheme. For example, this in principle allows for greater flexibility 
in the context of R-Marchenko windowing where different, time-asymmetric operators could be applied separately for up- and down-going pressure field components. We have begun to look into the implications of this observation, and will report on the outcome in future publications.

Given that the scattering schemes we present here make explicit use of a priori models with sharp contrasts included, it is natural to question the sensitivity of our approaches to errors in these models. Firstly, it is important to note that, even for the conventional Marchenko redatuming approach, in the case of complex media (e.g., subsalt) one requires the direct-arrival estimate to be as kinematically accurate as possible. In previous Marchenko studies in subsalt environments - which included field data (Jia et al., 2018; Staring et al., 2018; Staring and Wapenaar, 2020) - complex salt models were in fact used for the estimation of direct arrivals for the initial focusing functions. This is done because, in the presence of large contrasts such as salt, model smoothing can lead to significant phase distortions that affect direct-wave kinematics. This is not only true for Marchenko redatuming, but generally a well-known fact in imaging practice, e.g., in the case of reverse-time migration in subsalt environments. So, in that sense, here our approaches simply make better use of the same type of high-end models already in use for the purpose of general depth imaging as well as Marchenko redatuming. However, model errors do have an effect in Marchenko redatuming. In the context of the original Marchenko framework, previous studies (Broggini et al., 2014; Thorbecke et al., 2013) have shown that velocity model errors lead to image distortions similar to those in conventional migration, but that the handling of internal-multiples and their artifacts is rather robust with respect to relatively large velocity errors. In our case, because the benefits arising from the scattering schemes are rooted on complex wave events in $\mathbf{f}_{\mathbf{0}}$ - and those depend on the sharp impedance contrast in the model - we expect our 
methods to have greater sensitivity to model parameters than conventional Marchenko, and in particular to the geometry of sharp interfaces. Generally, however, we expect that current industry-standard models built to maximise migration image quality - i.e., to optimise focusing in subsurface angle gathers - in complex environments to be sufficiently accurate also for our redatuming purposes. Verifying this claim, as well as building a more thorough understanding of the role of model accuracy in our approaches is beyond the scope of this paper, but is the subject of current study.

One of our main goals with this work, particularly through the Scattering-Rayleigh scheme, is to enable Marchenko-driven redatuming and imaging applications in complex media that also handle band-limited data with realistic acquisition configurations. In a companion paper, Ravasi and Vasconcelos (2020a) present an open-source-based implementation of Marchenko operators for HPC environments - cluster or cloud-based - and demonstrate how such a framework enables research and development of approaches such as ours for 3D seismic data. The Pylops-based (Ravasi and Vasconcelos, 2020b) codes we developed and used in this study are already deployable under the HPC framework by Ravasi and Vasconcelos (2020a), as such, our approach is ready for validation with 3D field seismic data. At the time of writing this manuscript, we have recently been given access to a $3 \mathrm{D}$ ocean-bottom, permanent reservoir monitoring (PRM) dataset from the North Sea - which we will use as a testbed for validation and further research of the approaches herein.

As a final discussion point, we highlight that the improvements in waveform fidelity of redatumed fields in complex media brought by our scattering-based schemes bring new opportunities in the broader context of target-oriented inversion and monitoring. In the examples we provide here, Marchenko-redatumed fields are used for the estimation of localreflection extended images, and subsequently used for local imaging by means of depth 
migration - this is inline with, e.g., the Marchenko imaging steps as described by Wapenaar et al. (2014b). While this is one option for targeted imaging using Marchenko fields, it is by no means the only option. For example, when retrieved with sufficient waveform fidelity, Marchenko fields can be employed in local full-waveform inversion approaches (Vasconcelos et al., 2017; Cui et al., 2020; Elison et al., 2018). Alternatively, Marchenko fields may be integrated in other inversion schemes, such an in inverse-scattering-series-based (e.g., Guo and Alkhalifah, 2020), or directly into wave-equation solutions as a constraint (Diekmann and Vasconcelos, 2021; van Leeuwen and Herrmann, 2015). These being some of the uses of Marchenko fields in inverse schemes, they attest to the potential of such fields in contributing to target-oriented inversion.

Finally, though in this paper we focus on targeted imaging in complex media, the scattering formalism and approaches we present are equally suitable for time-lapse problems - where the reference medium and data are no longer dictated by a background model, but instead given in the form of the baseline model and observed data, respectively. The application of our scattering schemes to monitoring will be a focus in our upcoming field data studies in the context of PRM.

\section{CONCLUSION}

While the recent rise of redatuming and imaging approaches based on the Marchenko framework showed great promise for tackling the challenges of imaging in highly complex media, such as subsalt or sub-basalt, previous Machenko schemes impose limitations that can severely hinder accurate wavefield retrieval in such media. We present scattering-based approaches to Marchenko redatuming that can handle highly complex media, by taking advantage of high-end subsurface models typically built for migration. Our approaches take 
wavefield redatuming accuracy to a level of waveform fidelity in redatuming far beyond that of conventional imaging, resulting on extended image gathers and target-oriented images that are consequently highly accurate - thus realising the potential of the Marchenko framework for imaging of complex subsurface environments. To facilitate the deployment of our scheme to band-limited data, with realistic acquisition and free-surface conditions, we combine our scattering approach with the Rayleigh-Marchenko scheme into a new method that can produce accurate wavefields and images from offshore or ocean-bottom four-component datasets. We support our new approaches with numerical experiments using a highly-complex, dirty-salt 2D model. Given our robust theoretical and numerical constructs, we expect that our approaches will be useful for imaging and monitoring in challenging subsurface geological conditions.

\section{ACKNOWLEDGMENTS}

We are grateful to Haorui Peng, Leon Diekmann, Andreas Tataris, and Tristan van Leeuwen for insightful discussions that contributed to the work in this paper. IV and MR are thankful to Joost van der Neut, whose input contributed to the early development of our ideas on scattering-based Marchenko. We thank the sponsors of the Utrecht Consortium for Subsurface Imaging (UCSI) for their financial support. 


\section{APPENDIX A}

\section{LOCAL EXTENDED IMAGES BY TIME-DOMAIN INTERFEROMETRIC REDATUMING}

Extended images (EIs, Vasconcelos et al., 2010), $R^{\cup}\left(\mathbf{x}_{\mathbf{f}}^{\prime}, \mathbf{x}_{\mathbf{f}} ; \omega\right)$, are virtual reflectivity gathers at a target level $\partial \mathbb{D}_{\mathbf{i}}$ formed by the interaction of decomposed wavefields $P^{+}\left(\mathbf{x}_{\mathbf{s}}, \mathbf{x}_{\mathbf{f}} ; \omega\right)$ and $P^{-}\left(\mathbf{x}_{\mathbf{s}}, \mathbf{x}_{\mathbf{f}} ; \omega\right)$, connecting the focal point $\mathbf{x}_{\mathbf{f}}$ to a set of sources (or receivers) $\mathbf{x}_{\mathbf{s}}$ at the surface level. In the frequency domain, the multidimensional convolution process can be discretized as follows (van der Neut and Herrmann, 2012; Wapenaar et al., 2011):

$$
P^{-}\left(\mathbf{x}_{\mathbf{s}}, \mathbf{x}_{\mathbf{f}} ; \omega\right)=\int_{\partial \mathbb{D}_{\mathbf{f}}} P^{+}\left(\mathbf{x}_{\mathbf{s}}, \mathbf{x}_{\mathbf{f}}^{\prime} ; \omega\right) R^{\cup}\left(\mathbf{x}_{\mathbf{f}}^{\prime}, \mathbf{x}_{\mathbf{f}} ; \omega\right) d \mathbf{x}_{\mathbf{f}}^{\prime} \rightarrow \mathbf{P}^{-}=\mathbf{P}^{+} \mathbf{R}^{\cup}
$$

The associated normal equations are then written as $\mathbf{C}=\mathbf{\Gamma} \mathbf{R}^{\cup}$ (e.g. Vasconcelos and Rickett, 2013), where the point spread function $\Gamma$ is a blurring operator acting on $\mathbf{R}^{\cup}$ determining the crosscorrelation gather $\mathbf{C}$,

$$
\begin{aligned}
& C\left(\mathbf{x}_{\mathbf{f}}^{\prime}, \mathbf{x}_{\mathbf{f}} ; \omega\right)=\int_{\partial \mathbb{D}_{\mathbf{s}}} P^{+*}\left(\mathbf{x}_{\mathbf{f}}^{\prime}, \mathbf{x}_{\mathbf{s}} ; \omega\right) P^{-}\left(\mathbf{x}_{\mathbf{s}}, \mathbf{x}_{\mathbf{f}} ; \omega\right) d \mathbf{x}_{\mathbf{s}} \rightarrow \mathbf{C}=\mathbf{P}^{+\dagger} \mathbf{P}^{-} \\
& \Gamma\left(\mathbf{x}_{\mathbf{f}}^{\prime}, \mathbf{x}_{\mathbf{f}} ; \omega\right)=\int_{\partial \mathbb{D}_{\mathbf{s}}} P^{+*}\left(\mathbf{x}_{\mathbf{f}}^{\prime}, \mathbf{x}_{\mathbf{s}} ; \omega\right) P^{+}\left(\mathbf{x}_{\mathbf{s}}, \mathbf{x}_{\mathbf{f}} ; \omega\right) d \mathbf{x}_{\mathbf{s}} \rightarrow \boldsymbol{\Gamma}=\mathbf{P}^{+\dagger} \mathbf{P}^{+}
\end{aligned}
$$

here, it is important to note the use of the convention that discrete wavefield operators have sources on the row space and receivers on columns - this is opposite to the commonly used convention in MDD (e.g., Vasconcelos et al., 2010). The reason for this choice is that our numerical iterative solvers (e.g., LSQR), under the Pylops framework (Ravasi and Vasconcelos, 2020a,b), are designed to solve $\mathbf{A x}=\mathbf{y}$ for $\mathbf{x}$, assuming $\mathbf{A}$ is a left-hand operator - numerically this means we choose our row/column space convention so that the forward-operator fields are left-hand operators. 
A common approach to deconvolution in the frequency domain is to compute an inverse matrix for each frequency, $\mathbf{W}(\omega)=(\Gamma(\omega)+\epsilon \mathbf{I})^{-1}$, acting on the crosscorrelation gather, where $\epsilon$ aims at stabilizing the inversion and can be interpreted as a Tikhonov regularization term. In contrast, a time domain representation of the very same problem requires the definition of an operator $\hat{\mathbf{P}}^{+}=\mathbf{F}^{-\mathbf{1}} \mathbf{P}^{+} \mathbf{F}$ which acts on a vector and performs a step of multi-dimensional convolution (MDC) as described by Ravasi and Vasconcelos (2020b). Here $\mathbf{F}$ and $\mathbf{F}^{-\mathbf{1}}$, are forward and inverse space-time (2D) Fourier transform operators. While an explicit definition in computer memory of the equivalent matrix of such an operator is prohibitive, many iterative solvers only require the resulting forward and adjoint operations of the convolutional matrix operator on a given data vector - not an explicitly defined version of the operator in matrix form. With that in mind, using object-oriented representations of linear operators (Ravasi and Vasconcelos, 2020b) is an attractive alternative to solve large scale optimization problems in the time domain. Our implementation of multi-dimensional deconvolution in the time domain allows for the introduction of two preconditioners are can be written in the following way:

$$
\mathbf{p}^{-}=\left[\Theta \mathbf{F}^{-1} \mathbf{P}^{+} \mathbf{W F}\right] \mathbf{R}^{\cup}
$$

where $\mathbf{W}$ is a muting operator imposing frequency-wavenumber constraints, and $\boldsymbol{\Theta}$ is a muting operator in the time-space domain enforcing causality on the reflection response.

Since $\mathbf{C}$ is a blurred version of $\mathbf{R}^{\cup}$, it encompasses most of its data-space features in terms of the frequency-wavenumber support that can realistically be retrieved by MDD on a shot-by-shot basis. Consequently, the $f k$-domain spectrum of the crosscorrelation gathers are used to design a natural preconditioner, $\mathbf{W}$, constraining the inversion within the expected frequency-wavenumber support of $\mathbf{C}$. In the time-domain, $\boldsymbol{\Theta}$ has two roles: (1) 
it ensures the reflectivity responses from MDD are strictly non-negative, and (2) it enforces wave-equation causality by annihilating contributions arriving before direct-waves (based on the a priori migration model). Our experience shows that such preconditioning is essential to the retrieval of numerically robust, physically reliable, extended image/virtual source gathers by time-domain MDD. 


\section{REFERENCES}

Amundsen, L., 2001, Elimination of free-surface related multiples without need of the source wavelet: Geophysics, 66, 327-341.

Arasanipalai, S., H. Lebit, P. Ollagnon, B. Virlouvet, and J. Tilton, 2019, Enhanced presalt imaging using iterative least-squares migration: A case study in Santos Basin, Brazil: 89th Annual International Meeting, SEG, Expanded Abstracts, 4216-4220.

Baysal, E., D. D. Kosloff, and J. W. C. Sherwood, 1983, Reverse time migration: Geophysics, 48, 1514-1524.

Beck, A., and M. Teboulle, 2009, A fast iterative shrinkage-thresholding algorithm for linear inverse problems: SIAM Journal on Imaging Sciences, 2, 183-202.

Broggini, F., R. Snieder, and K. Wapenaar, 2014, Data-driven wavefield focusing and imaging with multidimensional deconvolution: Numerical examples for reflection data with internal multiples: Geophysics, 79, no. 3, WA107-WA115.

Cui, T., J. Rickett, I. Vasconcelos, and B. Veitch, 2020, Target-oriented full-waveform inversion using Marchenko redatumed wavefields: Geophysical Journal International, 223, $792-810$.

da Costa, C. A. N., J. Costa, W. E. Medeiros, D. J. Verschuur, and A. K. Soni, 2019, Targetlevel waveform inversion: a prospective application of the convolution-type representation for the acoustic wavefield: Geophysical Prospecting, 67, 69-84.

da Costa Filho, C. A., G. A. Meles, A. Curtis, M. Ravasi, and A. Kritski, 2017, Imaging strategies using focusing functions with applications to a North Sea field: Geophysical Journal International, 213, 561-573.

Diekmann, L., and I. Vasconcelos, 2021, Focusing and Green's function retrieval in threedimensional inverse scattering revisited: A single-sided Marchenko integral for the full 
wave field: Physical Review Research, 3, no. 1, 013206.

Dragoset, B., E. Verschuur, I. Moore, and R. Bisley, 2010, A perspective on 3D surface-related multiple elimination: Geophysics, 75, no. 5, 75A245-75A261.

Dukalski, M., and K. de Vos, 2017, Marchenko inversion in a strong scattering regime including surface-related multiples: Geophysical Journal International, 212, 760-776.

Dukalski, M., E. Mariani, and K. de Vos, 2019, Handling short-period scattering using augmented Marchenko autofocusing: Geophysical Journal International, 216, 2129-2133.

Elison, P., D. van Manen, J. Robertsson, M. Dukalski, and K. de Vos, 2018, Marchenko-based immersive wave simulation: Geophysical Journal International, 215, 1118-1131.

Esser, E., L. Guasch, F. J. Herrmann, and M. Warner, 2016, Constrained waveform inversion for automatic salt flooding: The Leading Edge, 35, 235-239.

Farmer, P., Z. Z. Zhou, and D. Jones, 2009, The role of reverse time migration in imaging and model estimation: The Leading Edge, 28, 436-441.

Fletcher, R. P., D. Nichols, R. Bloor, and R. T. Coates, 2016, Least-squares migration data domain versus image domain using point spread functions: The Leading Edge, 35, $157-162$.

Guo, Q., and T. Alkhalifah, 2020, Target-oriented waveform redatuming and high-resolution inversion: Role of the overburden: Geophysics, 85, no. 6, R525-R536.

Haindl, C., M. Ravasi, and F. Broggini, 2021, Handling gaps in acquisition geometries improving Marchenko-based imaging using sparsity-promoting inversion and joint inversion of time-lapse data: Geophysics, 0, 1-49.

Hennenfent, G., and F. J. Herrmann, 2008, Simply denoise: Wavefield reconstruction via jittered undersampling: Geophysics, 73, no. 3, V19-V28.

Jia, X., A. Guitton, and R. Snieder, 2018, A practical implementation of subsalt Marchenko 
imaging with a gulf of mexico data set: Geophysics, 83, no. 5, S409-S419.

Jones, I. F., and I. Davison, 2014, Seismic imaging in and around salt bodies: Interpretation, 2, SL1-SL20.

Leveille, J. P., I. F. Jones, Z.-Z. Zhou, B. Wang, and F. Liu, 2011, Subsalt imaging for exploration, production, and development: Geophysics, 76, no. 5, WB3-WB20.

Liu, Y., H. Hu, X.-B. Xie, Y. Zheng, and P. Li, 2015, Reverse time migration of internal multiples for subsalt imaging: Geophysics, 80, no. 5, S175-S185.

Malcolm, A. E., B. Ursin, and M. V. de Hoop, 2008, Subsalt imaging with internal multiples: 78th Annual International Meeting, SEG, Expanded Abstracts, 2461-2465.

McMechan, G. A., 1983, Migration by extrapolation of time-dependent boundary values: Geophysical Prospecting, 31, 413-420.

Mildner, C., F. Broggini, C. A. da Costa Filho, and J. O. Robertsson, 2019a, Source wavelet correction for practical Marchenko imaging: A sub-salt field-data example from the Gulf of Mexico: Geophysical Prospecting, 67, 2085-2103.

Mildner, C., F. Broggini, K. de Vos, and J. O. A. Robertsson, 2019b, Accurate source wavelet estimation using Marchenko focusing functions: Geophysics, 84, no. 6, Q73-Q88.

Nemeth, T., C. Wu, and G. T. Schuster, 1999, Least-squares migration of incomplete reflection data: Geophysics, 64, 208-221.

Paige, C. C., and M. A. Saunders, 1982, LSQR: An algorithm for sparse linear equations and sparse least squares: ACM Transactions on Mathematical Software, 8, 43-71.

Ravasi, M., 2017, Rayleigh-Marchenko redatuming for target-oriented, true-amplitude imaging: Geophysics, 86, no. 6, S439-S452.

Ravasi, M., and I. Vasconcelos, 2020a, On the implementation of large-scale integral operators with modern HPC solutions - application to 3D Marchenko imaging by least-squares 
inversion.

— $2020 \mathrm{~b}$, PyLops - A linear-operator Python library for scalable algebra and optimization: SoftwareX, 11, 100361.

Ravasi, M., I. Vasconcelos, and A. Curtis, 2014, Beyond conventional migration: non-linear elastic subsalt imaging with transmissions and two-sided illumination: Geophysical Journal International, 198, 1173-1185.

Ravasi, M., I. Vasconcelos, A. Kritski, A. Curtis, C. A. d. C. Filho, and G. A. Meles, 2016, Target-oriented Marchenko imaging of a North Sea field: Geophysical Journal International, 205, 99-104.

Slob, E., and K. Wapenaar, 2017, Theory for Marchenko imaging of marine seismic data with free surface multiple elimination: 79th Annual International Conference and Exhibition, EAGE, Extended Abstracts, 1-5.

Staring, M., R. Pereira, H. Douma, J. van der Neut, and K. Wapenaar, 2018, Sourcereceiver Marchenko redatuming on field data using an adaptive double-focusing method: Geophysics, 83, no. 6, S579-S590.

Staring, M., and K. Wapenaar, 2020, Three-dimensional Marchenko internal multiple attenuation on narrow azimuth streamer data of the Santos Basin, Brazil: Geophysical Prospecting, 68, 1864-1877.

Tarantola, A., 1984, Inversion of seismic reflection data in the acoustic approximation: Geophysics, 49, 1259-1266.

Thorbecke, J., J. van der Neut, and K. Wapenaar, 2013, Green's function retrieval with Marchenko equations: A sensitivity analysis: 83th Annual International Meeting, SEG, Expanded Abstracts, 3888-3893.

van Borselen, R. G., J. T. Fokkema, and P. M. van den Berg, 1996, Removal of surface-related 
wave phenomena: The marine case: Geophysics, 61, 202-210.

van den Berg, E., and M. P. Friedlander, 2009, Probing the Pareto frontier for basis pursuit solutions: SIAM Journal on Scientific Computing, 31, 890-912.

van der Neut, J., and F. J. Herrmann, 2012, Interferometric redatuming by sparse inversion: Geophysical Journal International, 192, 666-670.

van der Neut, J., J. Thorbecke, K. Wapenaar, and E. Slob, 2015a, Inversion of the multidimensional Marchenko equation: 77th Annual International Conference and Exhibition, EAGE, Extended Abstracts, 1-5.

van der Neut, J., I. Vasconcelos, and K. Wapenaar, 2015b, On Green's function retrieval by iterative substitution of the coupled Marchenko equations: Geophysical Journal International, 203, 792-813.

van der Neut, J., K. Wapenaar, J. Thorbecke, and E. Slob, 2015c, Practical challenges in adaptive Marchenko imaging: 85th Annual International Meeting, SEG, Expanded Abstracts, 4505-4509.

van Leeuwen, T., and F. J. Herrmann, 2015, A penalty method for PDE-constrained optimization in inverse problems: Inverse Problems, 32, 015007.

Vasconcelos, I., M. Ravasi, and J. van der Neut, 2017, Subsurface-domain, interferometric objective functions for target-oriented waveform inversion: Geophysics, 82, no. 4, A37A41.

Vasconcelos, I., and J. Rickett, 2013, Broadband extended images by joint inversion of multiple blended wavefields: Geophysics, 78, no. 2, WA147-WA158.

Vasconcelos, I., P. Sava, and H. Douma, 2010, Nonlinear extended images via image-domain interferometry: Geophysics, 75, no. 6, SA105-SA115.

Vasconcelos, I., and Y. Sripanich, 2019, Scattering-based Marchenko for subsurface focusing 
and redatuming in highly complex media: 81st Annual International Conference and Exhibition, EAGE, Extended Abstracts, 1-5.

Vasconcelos, I., D.-J. van Manen, M. Ravasi, K. Wapenaar, and J. van der Neut, 2014, Marchenko redatuming: Advantages and limitations in complex media: Presented at the 84th Annual International Meeting, SEG, Expanded Abstracts.

Verschuur, D. J., A. J. Berkhout, and C. P. A. Wapenaar, 1992, Adaptive surface-related multiple elimination: Geophysics, 57, 1166-1177.

Virieux, J., and S. Operto, 2009, An overview of full-waveform inversion in exploration geophysics: Geophysics, 74, WCC1-WCC26.

Wang, P., Z. Zhang, J. Mei, F. Lin, and R. Huang, 2019, Full-waveform inversion for salt: A coming of age: The Leading Edge, 38, 204-213.

Wapenaar, C. P. A., and J. L. T. Grimbergen, 1996, Reciprocity theorems for one-way wavefields: Geophysics, 127, 169-177.

Wapenaar, K., 1998, Reciprocity properties of one-way propagators: Geophysics, 63, 17951798.

Wapenaar, K., E. Slob, and R. Snieder, 2008, Seismic and electromagnetic controlled-source interferometry in dissipative media: Geophysical Prospecting, 56, 419-434.

Wapenaar, K., J. Thorbecke, and D. Draganov, 2004, Relations between reflection and transmission responses of three-dimensional inhomogeneous media: Geophysical Journal International, 156, 179-194.

Wapenaar, K., J. Thorbecke, J. van der Neut, F. Broggini, E. Slob, and R. Snieder, 2014a, Green's function retrieval from reflection data, in absence of a receiver at the virtual source position: The Journal of the Acoustical Society of America, 135, 2847-2861.

——, 2014b, Marchenko imaging: Geophysics, 79, no. 3, WA39-WA57. 
Wapenaar, K., J. van der Neut, E. Ruigrok, D. Draganov, J. Hunziker, E. Slob, J. Thorbecke, and R. Snieder, 2011, Seismic interferometry by crosscorrelation and by multidimensional deconvolution: A systematic comparison: Geophysical Journal International, 185, 13351364. 


\section{LIST OF FIGURES}

1 Scattering-based Marchenko configuration. The reference model (a) provides additional information for Marchenko redatuming by introducing the full transmission $\mathbf{g}_{0}$ and reflection response $\mathbf{R}_{0}$. For the true medium (b), the S-Marchenko representation considers a transparent surface $\partial \mathbb{D}_{0}$ in contrast to that in the SR-Marchenko (c) where the wavefield $\mathbf{V}_{\mathbf{z}}^{+}$experiment multiple reflections with the surface $\partial \mathbb{D}_{s}$. Focusing functions in all cases are defined in a truncated medium between levels $\partial \mathbb{D}_{0}$ and $\partial \mathbb{D}_{i}$.

2 Velocity models used in the sub-salt wavefield focusing and redatuming synthetic examples. (a) The truncated velocity model showing the overburden's shape laying on a smooth background resembling conventional migration velocity models. The true medium, (b), exhibits a complex heterogeneous overburden polluted with randomly distributed diffractors (dirty salt). At $4.4 \mathrm{~km}$, a target medium is selected for imaging beneath the salt's body enclosed by the dashed line. The solid blue dots represent virtual receiver positions at $\mathbf{x}_{\mathbf{f}}=(4.4,8.13) \mathrm{km}$, and $\mathbf{x}_{\mathbf{f}}=(4.4,6.0) \mathrm{km}$, respectively.

3 Scattering Marchenko wavefields. In the top row are total focusing functions for the reference model (a), perturbations relative to the true media (b), and the final focusing field in the true media, $\mathbf{f}=\mathbf{f}_{\mathbf{0}}+\mathbf{d f}$ (c). We point out the presence of physical contributions both within and outside the muting window in $\mathbf{f}_{\mathbf{0}}$ - these a key role in our scattering scheme. The bottom row shows corresponding Green's function reconstructions using focusing functions $\mathbf{f}_{\mathbf{0}}, \mathbf{d f}$, and $\mathbf{f}$. Most of the information from the deeper part of the media not resolved in the reference transmission (d) is recovered in the perturbed Green's function (e).

4 Different focusing functions for the virtual point located at $\mathbf{x}_{\mathbf{f}}=(4.4,8.13) \mathrm{km}$. (a) corresponds to the result estimated by S-Marchenko, and (b) the conventional focusing scheme. In panel (c), a close-up view by trace comparison indicates some of the most prominent features where events 
are underestimated or missing. Matching traces are extracted from a receiver at $8.8 \mathrm{~km}$ (colored lines in the gathers).

5 Comparisons between the forward modeled pressure field (a) and estimates from the SMarchenko (b) and the original Marchenko (c). The total field is the superposition of retrieved upand downgoing fields, given by $\mathbf{g}=\mathbf{g}^{-}+\mathbf{g}^{+}$. All panels are displayed with the same amplitude scaling. (d) displays corresponding traces from a receiver at $9.2 \mathrm{~km}$, uncovering phase and amplitude differences.

$6 \quad$ Scattering-Rayleigh Marchenko wavefields. Inverted focusing operator using the migration velocity model (a), followed by perturbations (b) providing additional refinements to produce the final total focusing function (c). The associated pressure fields are retrieved from band-limited data containing all internal and surface-related multiples. (d) corresponds to the background pressure fields, (e) to the reconstructed scattered result, and (f) is the total redatumed pressure response.

$7 \quad$ Focusing functions on band-limited data for a focal point at $\mathbf{x}_{\mathbf{f}}=(4.4,6.0) \mathrm{km}$. (a) the solution of our two-step focusing process with SR-Marchenko scheme, and (b) conventional R-Marchenko focusing. Discrepancies arise as a consequence of the different roles of the muting window in both methods as observed in the trace comparison (c) extracted from (a), (b) at corresponding space-time locations (colored lines in the gathers).

8 (a) The directly modeled pressure for evaluation of the (b) SR-Marchenko and (c) Marchenko retrieved pressure response for a fixed virtual source $\mathbf{x}_{\mathbf{f}}=(4.4,6.0) \mathrm{km}$ and variable receiver position. (d) An overlay of traces demonstrates a better amplitude prediction of the scattering-driven technique (red-dashed line) when compared with the R-Marchenko (blue-dashed line) against the numerically modeled solution (solid black line).

$9 \quad$ Local extended images for a virtual source at $\mathbf{x}_{\mathbf{f}}=(4.4,7.5) \mathrm{km}$. (a) For comparison, a benchmark, finite-difference modeled pressure field up to $40 \mathrm{~Hz}$ in a truncated section of the model, 
Figure 2, below the focusing datum at $4.4 \mathrm{~km}$. (b) S-Marchenko-based reflectivity. (c) Marchenko driven reflection response. (d) corresponds to a band-limited directly modeled reflection response up to $20 \mathrm{~Hz}$. (e) and (f) are reconstructed counterparts using the SR-Marchenko and R-Marchenko redatumed wavefields. Most of the major events in (a) and (d) recovered in (b) and (e) are not present in the (c) and (f). Similarly, substantial discontinuities and missing events arise in (c) and (f), contrary to (b) and (e).

10 Target-medium RTM images from the extended image/virtual gathers retrieved by MDD. For reference, (a) The migrated image of the local numerically modeled reflection response (benchmark), (b) the corresponding image from the S-Marchenko-driven redatumed response, and (c) the RTM image after conventional Marchenko. Boxes 1, 2, and 3 highlight areas for the close-ups in Figure 12.

11 (a) The RTM image from the synthetic data generated with finite differences, (b) migrated image from band-limited SR-Marchenko gathers, and (c) structural image from the migration of conventional R-Marchenko virtual gathers. Close-up images of boxes 1, 2, and 3 are depicted in Figure 13.

12 RTM zoomed sections associated with boxes 1, 2, and 3 in Figure 10. Columns (a), (b), (c) correspond to migrated images from the synthetic, S-Marchenko, and Marchenko virtual gathers accordingly. The top row corresponds to the green box 1, the middle row linked to the magenta box 2 , and the bottom row with that of the cyan box 3 .

13 RTM close-up sections extracted from boxes 1, 2, and 3 in Figure 11. The top row corresponds to the green box 1 , the middle row coupled to the magenta box 2 , and the bottom row to that of the cyan box 3. Columns (a), (b), (c) correspond to migrated images from the synthetic, SR-Marchenko, and R-Marchenko virtual gathers, respectively. 
a)

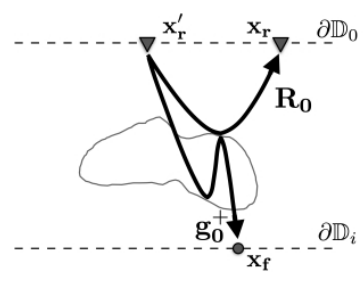

b)

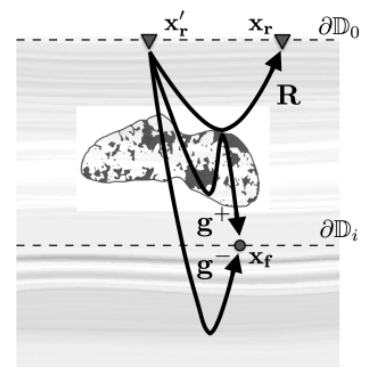

c)

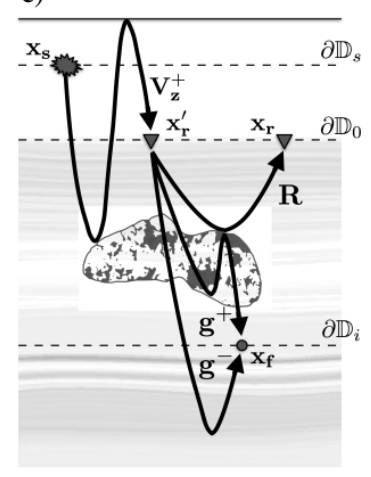

Figure 1: Scattering-based Marchenko configuration. The reference model (a) provides additional information for Marchenko redatuming by introducing the full transmission $\mathbf{g}_{\mathbf{0}}$ and reflection response $\mathbf{R}_{\mathbf{0}}$.

For the true medium (b), the S-Marchenko representation considers a transparent surface \$\partial Imathbb $\{\mathrm{D}\}_{-}\{0\} \$$ in contrast to that in the SR-Marchenko (c) where the wavefield $\$ \backslash \operatorname{mathbf}\left\{\mathrm{V}_{-}\{\mathrm{z}\} \wedge\{+\}\right\} \$$ experiment multiple reflections with the surface $\$ \backslash$ partial $\backslash$ mathbb $\{D\} \_\{s\} \$$. Focusing functions in all cases are defined in a truncated medium between levels $\$ \backslash$ partial \mathbb $\{D\}_{-}\{0\} \$$ and $\$ \backslash$ partial $\backslash$ mathbb $\{\mathrm{D}\} \_\{\mathrm{i}\} \$$. 
a)

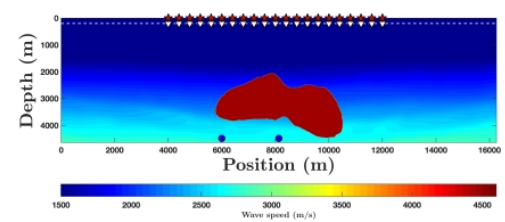

b)

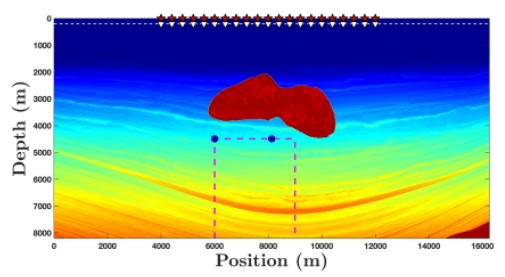

Figure 2: Velocity models used in the sub-salt wavefield focusing and redatuming synthetic examples. (a) The truncated velocity model showing the overburden's shape laying on a smooth background resembling conventional migration velocity models. The true medium, (b), exhibits a complex heterogeneous overburden polluted with randomly distributed diffractors (dirty salt). At $4.4 \mathrm{~km}$, a target medium is selected for imaging beneath the salt's body enclosed by the dashed line. The solid blue dots represent virtual receiver positions at $\mathbf{x}_{\mathbf{f}}=(4.4,8.13) \mathrm{km}$, and $\mathbf{x}_{\mathbf{f}}=(4.4,6.0) \mathrm{km}$, respectively. 
a)

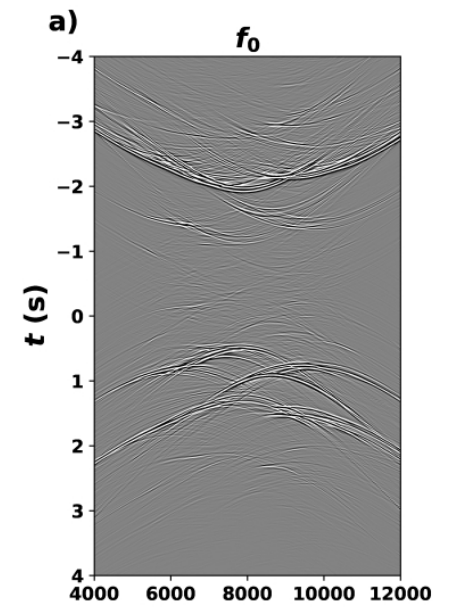

d)

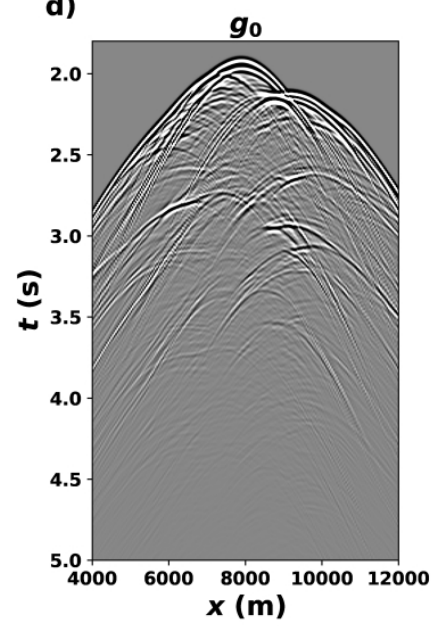

b)

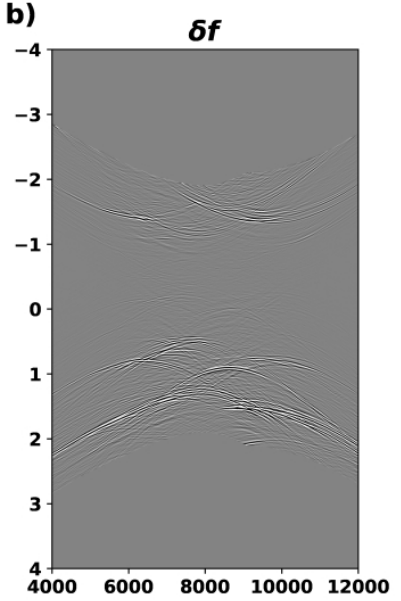

e)

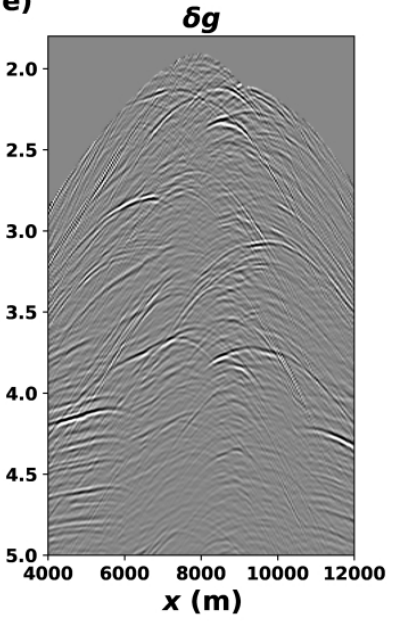

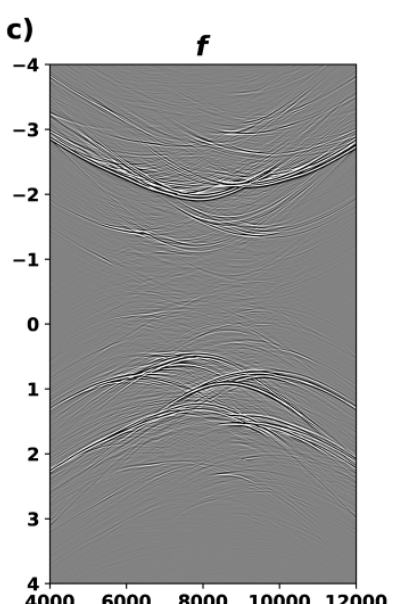

f)

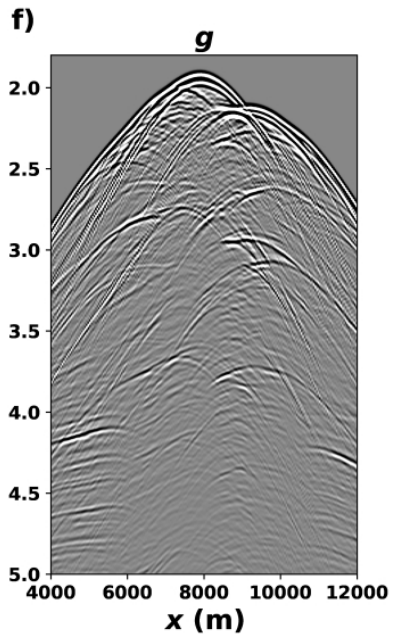

Figure 3: Scattering Marchenko wavefields. In the top row are total focusing functions for the reference model (a), perturbations relative to the true media (b), and the final focusing field in the true media, $\$ \backslash \operatorname{mathbf}\{\mathrm{f}\}=\backslash \operatorname{mathbf}\left\{\mathrm{f}_{-}\{0\}\right\}+\backslash \operatorname{mathbf}\{\mathrm{df}\} \$(\mathrm{c})$. We point out the presence of physical contributions both within and outside the muting window in $\$ \backslash$ mathbf $\{$ f_0 0$\} \$$ - these a key role in our scattering scheme.

The bottom row shows corresponding Green's function reconstructions using focusing functions $\$ \backslash \operatorname{mathbf}\left\{f_{-}\{0\}\right\} \$, \$ \backslash \operatorname{mathbf}\{\mathrm{df}\} \$$, and $\$ \backslash \operatorname{mathbf}\{\mathrm{f}\} \$$. Most of the information from the deeper part of the media not resolved in the reference transmission (d) is recovered in the perturbed Green's function (e). 
a)

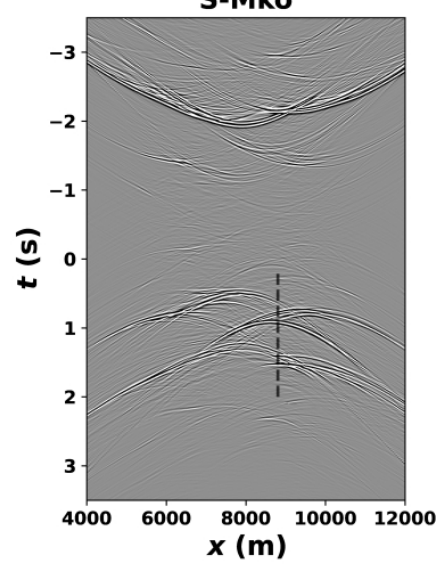

b)

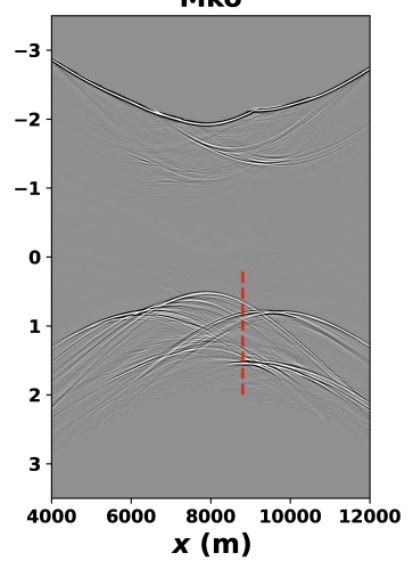

c)

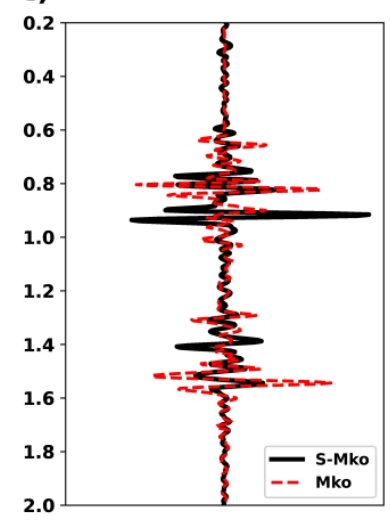

Figure 4: Different focusing functions for the virtual point located at $\mathbf{x}_{\mathbf{f}}=(4.4,8.13) \mathrm{km}$. (a) corresponds to the result estimated by S-Marchenko, and (b) the conventional focusing scheme. In panel (c), a close-up view by trace comparison indicates some of the most prominent features where events are underestimated or missing. Matching traces are extracted from a receiver at $8.8 \mathrm{~km}$ (colored lines in the gathers). 

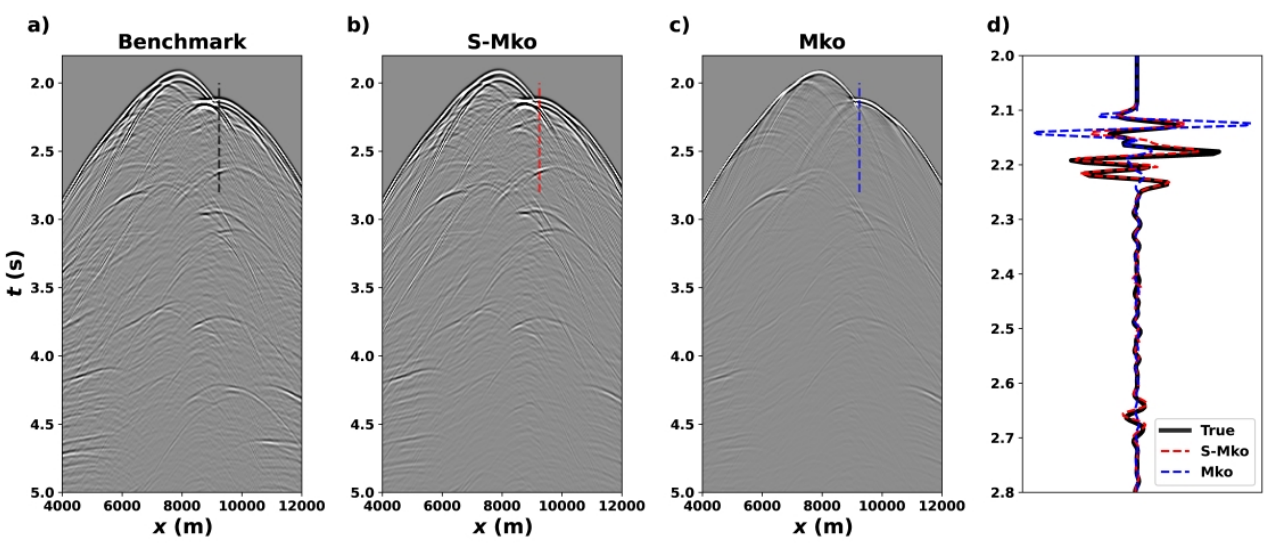

Figure 5: Comparisons between the forward modeled pressure field (a) and estimates from the S-Marchenko (b) and the original Marchenko (c). The total field is the superposition of retrieved up- and downgoing fields, given by $\$ \backslash \operatorname{mathbf}\{\mathrm{g}\}=\mid \operatorname{mathbf}\{\mathrm{g}\}^{\wedge}\{-\}+\backslash \operatorname{mathbf}\{\mathrm{g}\}^{\wedge}\{+\} \$$. All panels are displayed with the same amplitude scaling. (d) displays corresponding traces from a receiver at $9.2 \mathrm{~km}$, uncovering phase and amplitude differences. 
a)

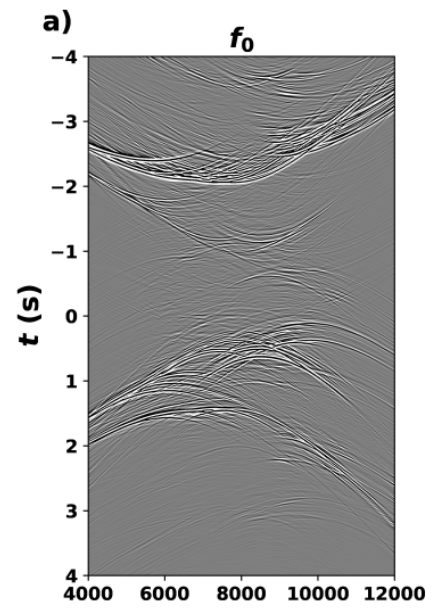

d)

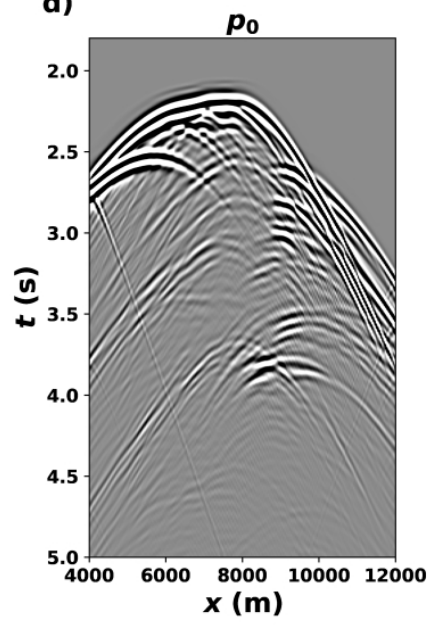

b)

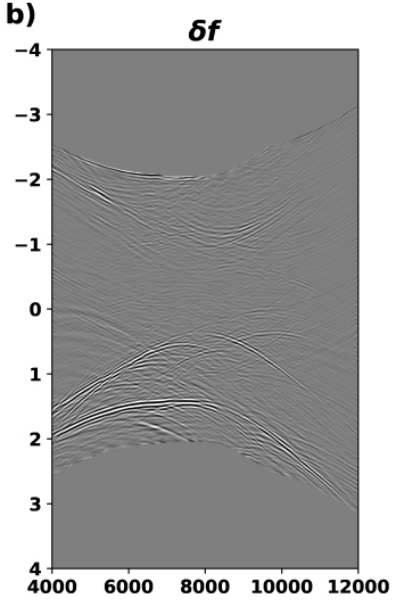

e)

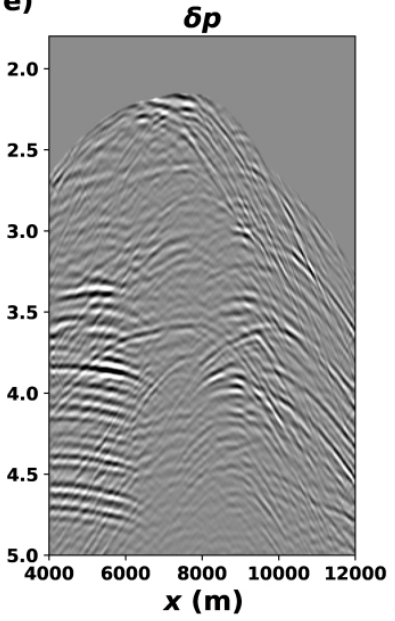

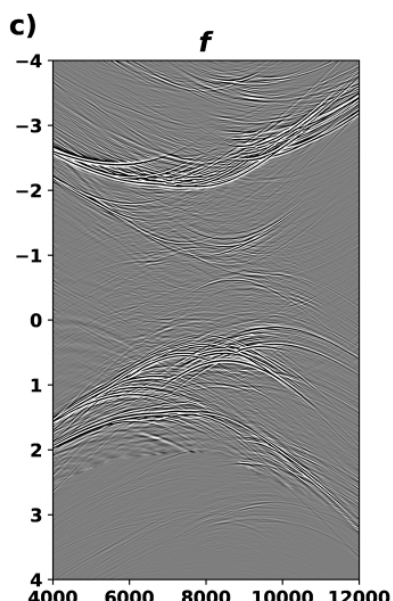

f)

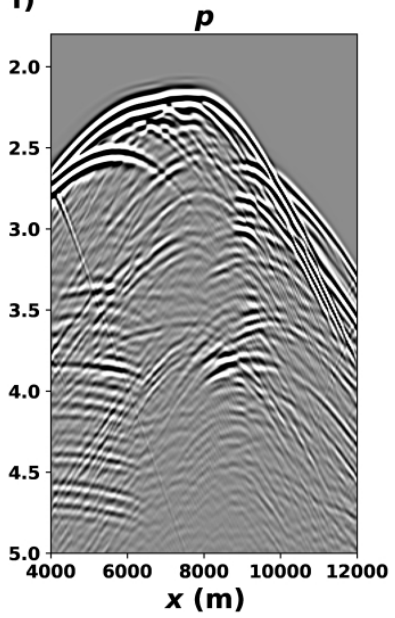

Figure 6: Scattering-Rayleigh Marchenko wavefields. Inverted focusing operator using the migration velocity model (a), followed by perturbations (b) providing additional refinements to produce the final total focusing function (c). The associated pressure fields are retrieved from band-limited data containing all internal and surface-related multiples. (d) corresponds to the background pressure fields, (e) to the reconstructed scattered result, and ( $f$ ) is the total redatumed pressure response. 
a)

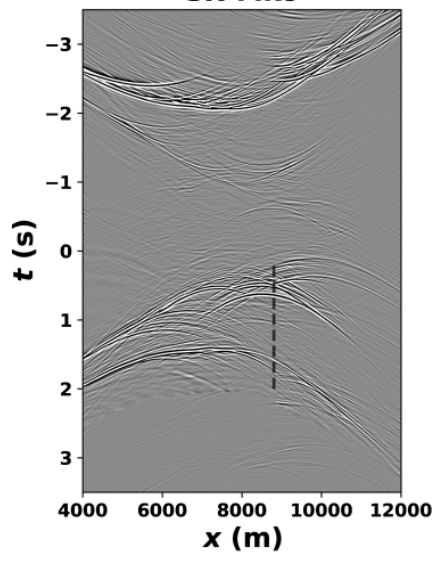

b)

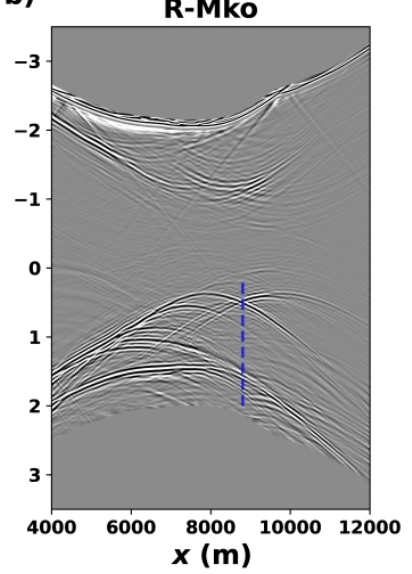

c)

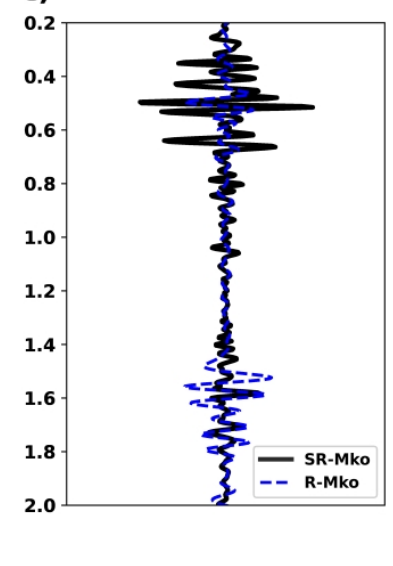

Figure 7: Focusing functions on band-limited data for a focal point at $\mathbf{x}_{\mathbf{f}}=(4.4,6.0) \mathrm{km}$. (a) the solution of our two-step focusing process with SR-Marchenko scheme, and (b) conventional R-Marchenko focusing. Discrepancies arise as a consequence of the different roles of the muting window in both methods as observed in the trace comparison (c) extracted from (a), (b) at corresponding space-time locations (colored lines in the gathers). 

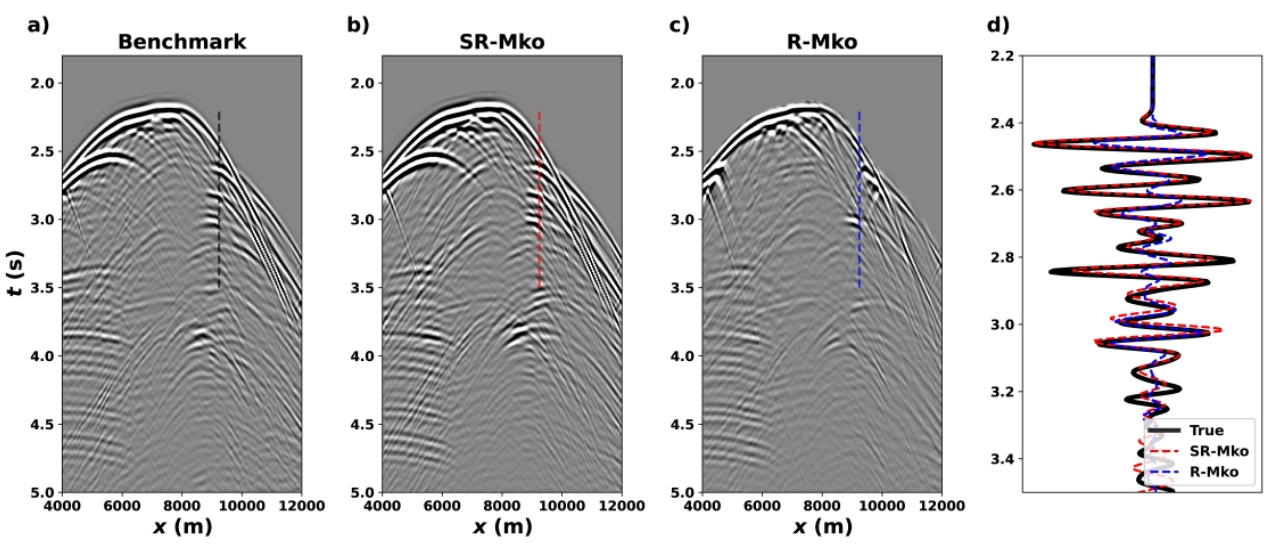

Figure 8: (a) The directly modeled pressure for evaluation of the (b) SR-Marchenko and (c) Marchenko retrieved pressure response for a fixed virtual source $\mathbf{x}_{\mathbf{f}}=(4.4,6.0) \mathrm{km}$ and variable receiver position. (d) An overlay of traces demonstrates a better amplitude prediction of the scattering-driven technique (reddashed line) when compared with the R-Marchenko (blue-dashed line) against the numerically modeled solution (solid black line). 

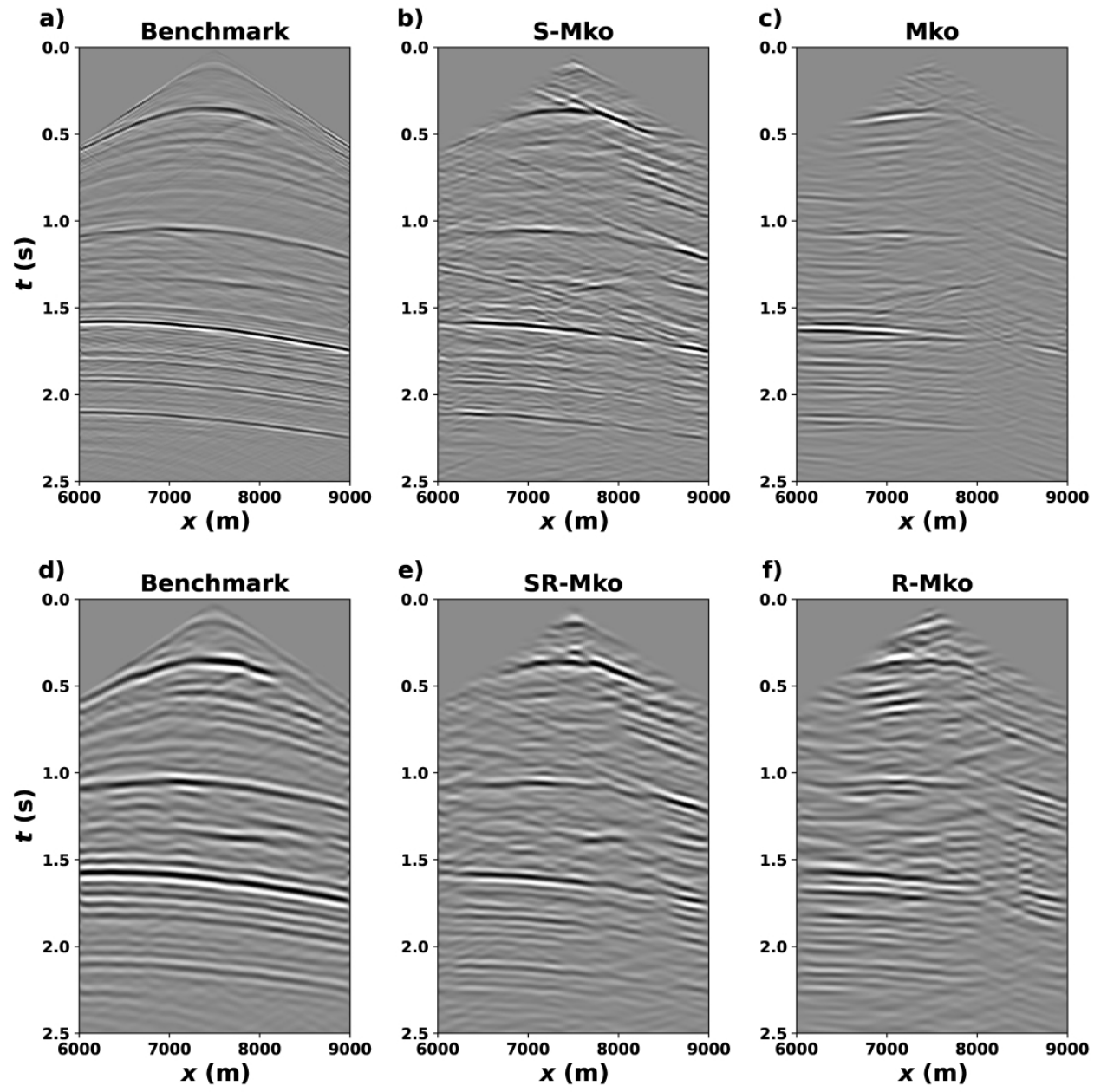

Figure 9: Local extended images for a virtual source at $\mathbf{x}_{\mathbf{f}}=(4.4,7.5) \mathrm{km}$. (a) For comparison, a benchmark, finite-difference modeled pressure field up to $40 \mathrm{~Hz}$ in a truncated section of the model, Figure 2, below the focusing datum at $4.4 \mathrm{~km}$. (b) S-Marchenko-based reflectivity. (c) Marchenko driven reflection response. (d) corresponds to a band-limited directly modeled reflection response up to $20 \mathrm{~Hz}$. (e) and (f) are reconstructed counterparts using the SR-Marchenko and R-Marchenko redatumed wavefields. Most of the major events in (a) and (d) recovered in (b) and (e) are not present in the (c) and (f). Similarly, substantial discontinuities and missing events arise in (c) and (f), contrary to (b) and (e). 
a)

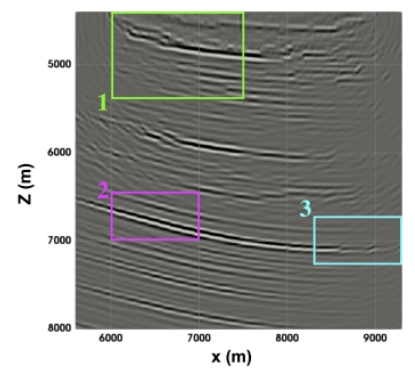

b)

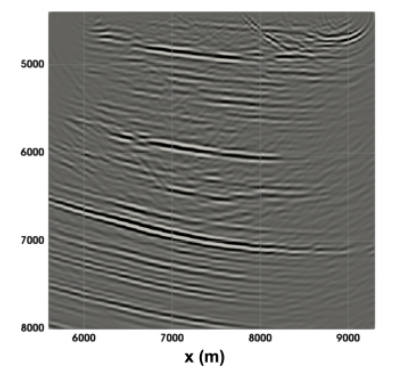

c)

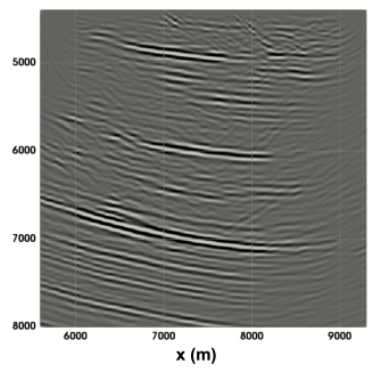

Figure 10: Target-medium RTM images from the extended image/virtual gathers retrieved by MDD. For reference, (a) The migrated image of the local numerically modeled reflection response (benchmark), (b) the corresponding image from the S-Marchenko-driven redatumed response, and (c) the RTM image after conventional Marchenko. Boxes 1, 2, and 3 highlight areas for the close-ups in Figure 12. 
a)

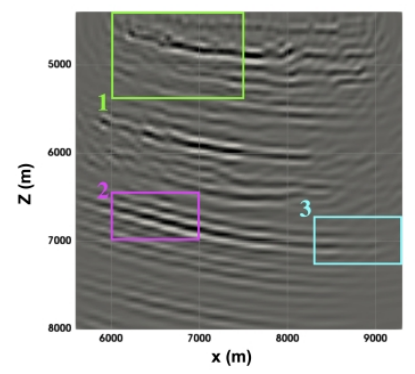

b)

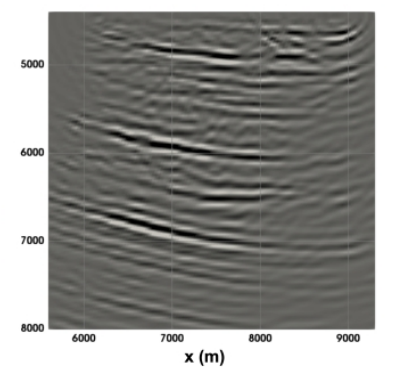

c)

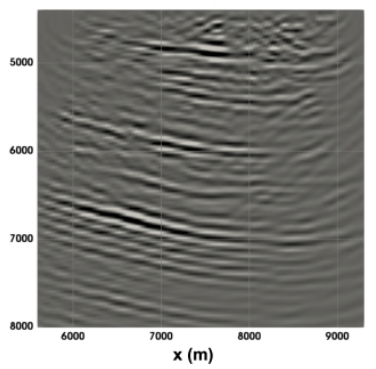

Figure 11: (a) The RTM image from the synthetic data generated with finite differences, (b) migrated image from band-limited SR-Marchenko gathers, and (c) structural image from the migration of conventional RMarchenko virtual gathers. Close-up images of boxes 1, 2, and 3 are depicted in Figure 13. 


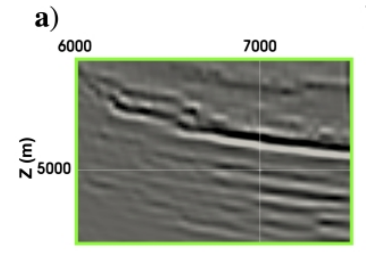

b)

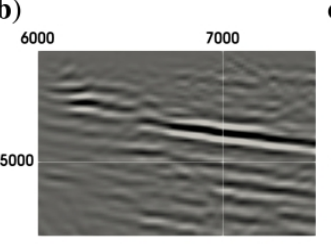

c)
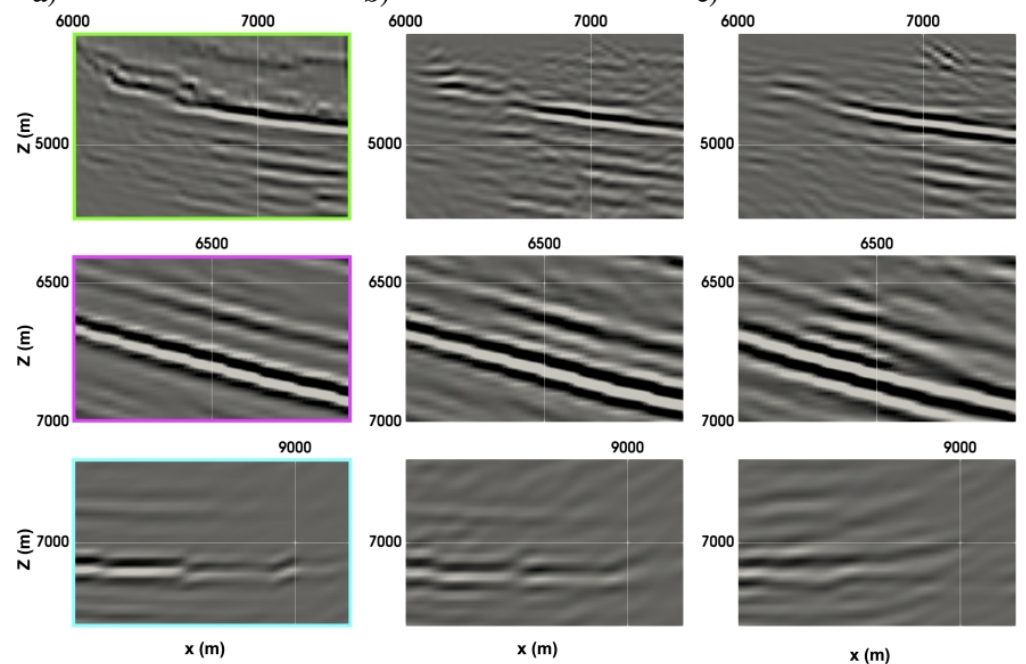

$x(m)$

$\mathbf{x}(\mathrm{m})$

Figure 12: RTM zoomed sections associated with boxes 1, 2, and 3 in Figure 10. Columns (a), (b), (c) correspond to migrated images from the synthetic, S-Marchenko, and Marchenko virtual gathers accordingly. The top row corresponds to the green box 1 , the middle row linked to the magenta box 2 , and the bottom row with that of the cyan box 3 . 


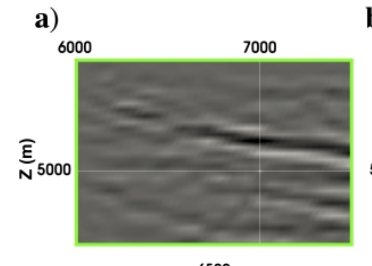

b)

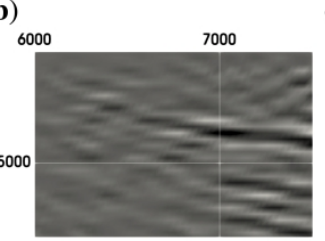

c)
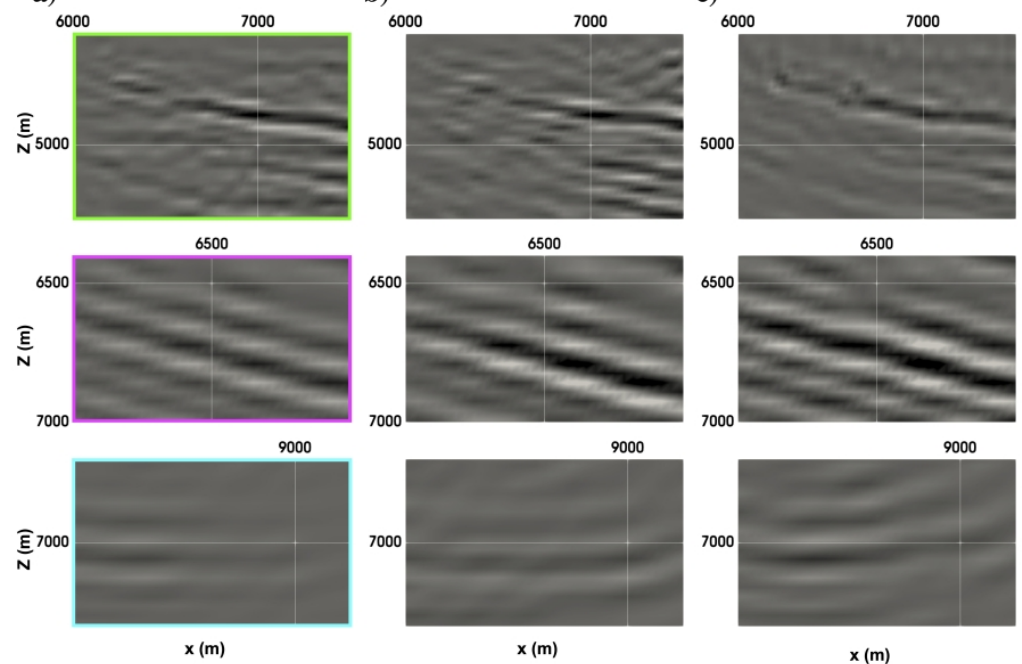

$x(m)$

Figure 13: RTM close-up sections extracted from boxes 1, 2, and 3 in Figure 11. The top row corresponds to the green box 1 , the middle row coupled to the magenta box 2, and the bottom row to that of the cyan box 3. Columns (a), (b), (c) correspond to migrated images from the synthetic, SR-Marchenko, and RMarchenko virtual gathers, respectively. 
DATA AND MATERIALS AVAILABILITY

Data associated with this research are available and can be obtained by contacting the corresponding author. 